\title{
The QCD Sign Problem for Small Chemical Potential
}

\author{
K. Splittorff ${ }^{1}$ and J.J.M. Verbaarschot ${ }^{1,2,3}$ \\ ${ }^{1}$ The Niels Bohr Institute, Blegdamsvej 17, DK-2100, Copenhagen Ø, Denmark \\ ${ }^{2}$ Niels Bohr International Academy, Blegdamsvej 17, DK-2100, Copenhagen Ø, Denmark \\ ${ }^{3}$ Department of Physics and Astronomy, SUNY, Stony Brook, New York 11794, USA
}

(Dated: July 5, 2018)

\begin{abstract}
The expectation value of the complex phase factor of the fermion determinant is computed in the microscopic domain of QCD at nonzero chemical potential. We find that the average phase factor is non-vanishing below a critical value of the chemical potential equal to the half the pion mass and vanishes exponentially in the volume for larger values of the chemical potential. This holds for QCD with dynamical quarks as well as for quenched and phase quenched QCD. The average phase factor has an essential singularity for zero chemical potential and cannot be obtained by analytic continuation from imaginary chemical potential or by means of a Taylor expansion. The leading order correction in the $p$-expansion of the chiral Lagrangian is calculated as well.
\end{abstract}




\section{INTRODUCTION}

A nonzero baryon density in QCD is achieved by introducing a chemical potential which enhances the propagation of quarks in the forward time direction and inhibits their propagation in the backward time direction. This imbalance makes the fermion determinant in the Euclidean formulation of QCD complex. The integration measure in the QCD partition function therefore cannot be used directly to define a probabilistic measure to generate gauge field configurations. Because of this sign problem, standard lattice QCD does not apply at nonzero baryon chemical potential. This does not mean that lattice QCD simulations at nonzero chemical potential are all altogether impossible. If the complex phase factor of the fermion determinant does not fluctuate strongly the sign problem may become manageable. The reweighting method [1] deals with the sign problem by including the phase factor in the observable rather than in the measure. While this method works in principle, its limitations are set by the strength of the fluctuations of the phase of the fermion determinant. It is therefore of considerable interest to understand how the fluctuations of the phase factor depend on the temperature, volume, chemical potential, quark mass as well as the lattice cutoff.

In this paper we study the severity of the sign problem in QCD as expressed through the vacuum expectation value of the phase factor

$$
e^{2 i \theta}=\frac{\operatorname{det}\left(D+\mu \gamma_{0}+m\right)}{\operatorname{det}\left(D^{\dagger}+\mu \gamma_{0}+m\right)} .
$$

Since the average phase factor is the ratio of two partition functions, it is typically exponentially small in the volume requiring that the number of configurations needed in a reweighting approach is exponentially large with the volume.

We will consider low temperatures where the chiral condensate $\Sigma$ is nonzero and pions dominate the excitations of the QCD vacuum. We will take the square of the chemical potential inversely proportional to the volume

$$
\mu^{2} F_{\pi}^{2} V \sim 1
$$

where $F_{\pi}$ is the pion decay constant. If furthermore

$$
m \Sigma V \sim 1 \text { and } V \Lambda_{\mathrm{QCD}}^{4} \gg 1,
$$

the QCD partition function reduces to the zero momentum limit of the corresponding chiral Lagrangian. The scaling of $\mu$ and $m$ in (2) and (3) defines the microscopic limit of QCD and is also referred to as the $\epsilon$-regime [2, 3]. In this limit, the zero momentum modes of the pions factorize from the QCD(-like) partition function and exact analytical expressions can be obtained. We will derive exact expressions for the average phase factor in both quenched QCD and QCD with dynamical quarks as well as in its phase quenched version. The result in all cases is that the expectation value of the phase factor is exponentially small in the volume when $\mu>m_{\pi} / 2$. For chemical potentials smaller than half the pion mass the average phase factor is nonzero. This suggests that the exact analytical predictions for $\mu<m_{\pi} / 2$ can be tested by means of lattice QCD even in the presence of dynamical quarks.

In the past few years several lattice QCD methods have been developed to study the properties of QCD at nonzero chemical potential. In particular, progress has been made with reweighting [4, 5], imaginary chemical potential [6, 7, [8] the Taylor expansion method [9, 10, 11, 12] and the density of states method [13] (for a recent review see [14]). Generally, one expects these methods to work if the average phase of the quark determinant fluctuates only mildly. For this reason several lattice measurements of the phase have appeared [13, 15, 16, 17, 18, 19, 20]. In this paper we will find that the quenched as well as the unquenched average of the phase factor has an essential singularity at zero chemical potential and cannot be obtained by analytic continuation from imaginary chemical potential or by means of a Taylor expansion. We give the explicit form of the non-analytic part and show that it is responsible for the abrupt change of the average phase factor at $\mu=m_{\pi} / 2$. We also give the exact predictions for purely imaginary $\mu$. Since these are analytic in $\mu$ they can be tested directly with simulations at imaginary $\mu$ or by means of Taylor expansion. We hope that the predictions for the average phase both for $\mu^{2}>0$ and for $\mu^{2}<0$ will motivate lattice studies of the phase fluctuations in the microscopic domain and thereby deepen our understanding of QCD at nonzero chemical potential.

The scale $\mu=m_{\pi} / 2$ has been part of lattice QCD at nonzero chemical potential starting from the very first calculations [20, 21] . Although it manifests itself in different ways in quenched and unquenched QCD, it has the same physical origin, namely condensation of charged pions.

The most direct effect of pion condensation occurs in QCD with an equal number of quarks and conjugate quarks (referred to as phase quenched QCD). For a conjugate quark, the fermion determinant is replaced by its complex conjugate, and therefore this theory corresponds to ignoring the phase factor of the fermion determinant. Since 
complex conjugation of a fermion determinant corresponds to changing the sign of the chemical potential [22], twoflavor phase quenched QCD is identical to QCD with a nonzero isospin chemical potential. Because the pions have a nonzero isospin charge and are weakly interacting they will Bose condense for $\mu>m_{\pi} / 2$ at zero temperature [23, 24, 25, 26].

The quenched approximation at nonzero chemical potential is the zero flavor limit of QCD with an equal number of quarks and conjugate quarks [27, 28]. It therefore inherits the scale $\mu=m_{\pi} / 2$. For example, one finds that the quark number density is nonzero for $\mu>m_{\pi} / 2$ [21, 29].

In QCD with dynamical quarks the Dirac spectrum for typical gauge field configurations has the same support as in the quenched case. Therefore, for quark masses such that $m_{\pi} / 2<\mu$, one or more of the quark masses are inside the domain of the Dirac eigenvalues, and the sign problem is severe. It has to be like that because the phase factor has to wipe out the Bose condensate of the phase quenched theory (see also the review [30]). In QCD with dynamical quarks, the scale $\mu=m_{\pi} / 2$ is thus the scale where phase fluctuations become strong. This is also illustrated by the spectral density of full QCD: When the quark mass is inside the support of the eigenvalues the unquenched spectral density becomes a complex function with strong oscillations in a finite domain of its support [31, 32. Physically these oscillations, with an amplitude that diverges exponentially with the volume and a period inversely proportional to the volume, are important because they are responsible for the discontinuity in the chiral condensate [33].

The difference between the phase quenched theory (where the phase of the fermion determinant is ignored) and unquenched QCD gives an indication of the importance of the phase factor. In fact the ratio of the unquenched partition function and the phase quenched partition function for two flavors is exactly the expectation value of the phase factor in the phase quenched theory (11). For $\mu<m_{\pi} / 2$ and zero temperature the free energy of the two partition functions is the same in the thermodynamic limit, but for $\mu>m_{\pi} / 2$, because of the formation of a Bose condensate in the phase quenched theory, the two free energies become different. This results in an exponential suppression of the average phase factor. In this paper we will show that the same behavior is found for the expectation value of the phase factor with respect to the full QCD partition function and the quenched QCD partition function. We will do this by an explicit calculation of the average phase factor in the microscopic domain of QCD.

In the microscopic limit QCD is equivalent to a chiral random matrix model with the same global symmetries [32, 34, 35, 36]. The reason is that the static part of the chiral Lagrangian is uniquely determined by global symmetries. We exploit this equivalence to calculate the average phase factor in this domain. Alternatively, we could have started from the static part of a chiral Lagrangian and performed the integral over the zero momentum modes of the Goldstone fields. Since the phase factor includes a bosonic determinant at nonzero chemical potential this method is, however, technically demanding and has not been worked out up to now.

In the microscopic limit it is useful to consider a fixed topological charge sector rather than a fixed vacuum angle [3]. For simplicity, in this paper we will only consider the sector of zero topological charge. The results for arbitrary topological charge follow by an immediate generalization.

Part of the results derived in this paper were announced in the letter [37]. In addition to providing details of the derivations, we obtain below explicit expressions for the average phase factor and show that it is a non-analytic function of the chemical potential. We also compute the corrections to the microscopic results to leading order in the $p$-expansion of the chiral Lagrangian.

The behavior of the average phase factor can be understood from general arguments presented in the next section. The connection between these general arguments and the exact expressions in the microscopic limit are explained in section [II where we analyze the average phase factor in the phase quenched theory. In sections [V and $\mathrm{V}$ we derive exact expressions for the average phase factor in the microscopic limit. The thermodynamic limit of these results is evaluated in section [VI, and the results obtained from general arguments will be confirmed. Section VII contains a discussion of the analytic properties of the average phase factor, and we finish with concluding remarks. Technical details are worked out in two appendices.

\section{THE AVERAGE PHASE FACTOR FROM A GENERAL ARGUMENT}

In this section we will consider the phase fluctuations of the fermion determinant in the QCD partition function

$$
Z_{N_{f}}=\left\langle\operatorname{det}^{N_{f}}\left(D+\mu \gamma_{0}+m\right)\right\rangle
$$

using mean field arguments at low energy. (Throughout this manuscript $\langle\ldots\rangle$ denotes the quenched average. Dynamical averages are labeled by a subscript referring to the number of flavors $N_{f}$.) In order to quantify the phase 
fluctuations of the fermion determinant we compute the average phase factor [54]

$$
\left\langle e^{2 i \theta}\right\rangle_{N_{f}}=\frac{1}{Z_{N_{f}}}\left\langle\frac{\operatorname{det}\left(D+\mu \gamma_{0}+m\right)}{\operatorname{det}\left(D^{\dagger}+\mu \gamma_{0}+m\right)} \operatorname{det}^{N_{f}}\left(D+\mu \gamma_{0}+m\right)\right\rangle .
$$

Notice that the average phase factor is the ratio of two partition functions: the partition function with an additional fermionic quark and an additional conjugate bosonic quark and the ordinary QCD partition function

$$
\left\langle e^{2 i \theta}\right\rangle_{N_{f}}=\frac{Z_{N_{f}+1 \mid 1^{*}}}{Z_{N_{f}}}
$$

This implies that the average phase factor is necessarily real. We will consider the low temperature limit with quark masses and chemical potentials well below $\Lambda_{\mathrm{QCD}}$. Then the partition function can be described in terms of a chiral Lagrangian. In this section we will focus on the mean field limit where the fields are replaced by their saddle point values. In this limit the partition functions assumes the general form

$$
Z \sim J\left(\prod_{k} \frac{1}{m_{\pi}(\mu)}\right) e^{-V F},
$$

where the Jacobian, $J$, is from the measure of the Goldstone manifold at the saddle point, and $m_{\pi}(\mu)$ are the $\mu$ dependent masses of the Goldstone modes. (For a discussion of the parameterization dependence of the masses of the Goldstone modes see Appendix $\mathrm{A}$ ) The free energy density at the saddle point, $F$, is intensive, and the prefactor can be written as a $1 / V$ correction to the free energy.

The QCD partition function, $Z_{N_{f}}$, in this approximation is completely independent of the chemical potential; since the pions have zero baryon charge neither the free energy density nor the exponential prefactor depend on $\mu$.

At zero chemical potential the fermion determinant is real so in this case the two additional determinants in $Z_{N_{f}+1 \mid 1^{*}}$ cancel and the average phase factor is 1 . When the chemical potential is nonzero, the two additional flavors become important. The combinations of the conjugate bosonic quark with any of the fermionic quarks forms a charged Goldstino. Because of the condensation of charged Goldstinos for $\mu>m_{\pi} / 2$ we need to consider separately the cases $\mu<m_{\pi} / 2$ and $\mu>m_{\pi} / 2$.

For $\mu<m_{\pi} / 2$ the free energy $F$ is independent of the chemical potential. In the expectation value of the phase factor the contributions of the free energy to the average phase cancel. The prefactor in (77) however depends on $\mu$ because the $\mu$-dependence of the masses of the charged Goldstone modes. Half of the $2\left(N_{f}+1\right)$ charged modes have masses $m_{\pi}(\mu)=m_{\pi}+2 \mu$ while the other half have masses $m_{\pi}(\mu)=m_{\pi}-2 \mu$. The mean field result for the ratio of $Z_{N_{f}+1 \mid 1^{*}}$ and $Z_{N_{f}}$ is thus given by

$$
\left\langle e^{2 i \theta}\right\rangle_{N_{f}}=\frac{\left(m_{\pi}-2 \mu\right)^{N_{f}+1}\left(m_{\pi}+2 \mu\right)^{N_{f}+1}}{m_{\pi}^{2 N_{f}+2}}=\left(1-\frac{4 \mu^{2}}{m_{\pi}^{2}}\right)^{N_{f}+1} \quad \text { for } \quad \mu<\frac{1}{2} m_{\pi} .
$$

For $2 \mu>m_{\pi}$ the free energy of $Z_{N_{f}+1 \mid 1^{*}}$ depends of $\mu$. The free energy has the usual $\mu$ dependence for the static part of a chiral Lagrangian in the condensed phase (see for example [23, 24, 26]). Subtracting the free energy at $\mu=0$ we find

$$
\left\langle e^{2 i \theta}\right\rangle_{N_{f}} \sim e^{-2 V F_{\pi}^{2} \mu^{2}\left(1-m_{\pi}^{2} / 4 \mu^{2}\right)^{2}} \quad \text { for } \quad \mu>\frac{1}{2} m_{\pi} .
$$

The average phase factor is thus suppressed exponentially with the volume for $\mu>m_{\pi} / 2$. Care has to be taken to compute the exponential prefactor. For $\mu>m_{\pi} / 2$ there are massless modes associated with the condensation of pions. Consequently the leading contribution to the prefactor given in (7) vanishes. One way to compute the subleading contributions to the prefactor is from the thermodynamic limit of the exact microscopic result. In the next section we perform this calculation in the phase quenched theory.

In section VI we will confirm the results obtained here from the thermodynamic limit of the exact microscopic expressions.

\section{THE AVERAGE PHASE FACTOR IN THE PHASE QUENCHED THEORY}

In this section we will discuss the chemical potential dependence of the average phase factor in the phase quenched theory, which can be easily obtained from known results. First we will discuss the exact microscopic result and then relate it to the mean field results of section 
The phase quenched partition function with two dynamical flavors is just the two-flavor QCD partition function without the phase of the fermion determinant,

$$
Z_{1+1^{*}}(m ; \mu)=\left\langle\left|\operatorname{det}\left(D+\mu \gamma_{0}+m\right)\right|^{2}\right\rangle .
$$

This partition function is equivalent to QCD at nonzero isospin chemical 22]. The expectation value of the phase factor of the fermion determinant for the phase quenched theory is thus given by

$$
\left\langle e^{2 i \theta}\right\rangle_{1+1^{*}}=\frac{\left\langle\operatorname{det}^{2}\left(D+\mu \gamma_{0}+m\right)\right\rangle}{\left\langle\left|\operatorname{det}\left(D+\mu \gamma_{0}+m\right)\right|^{2}\right\rangle}=\frac{Z_{N_{f}=2}}{Z_{1+1^{*}}} .
$$

Its microscopic limit therefore follows immediately from the microscopic limit of the two partition functions.

The microscopic limit of the phase quenched partition function is given by [38]

$$
Z_{1+1^{*}}(\hat{m} ; \hat{\mu})=2 e^{2 \hat{\mu}^{2}} \int_{0}^{1} d t t e^{-2 \hat{\mu}^{2} t^{2}} I_{0}^{2}(\hat{m} t)
$$

where

$$
\hat{m}=m V \Sigma \quad \text { and } \quad \hat{\mu}=\mu F_{\pi} \sqrt{V} .
$$

The two-flavor partition function is $\mu$-independent at scales well below the nucleon mass. Its microscopic limit is given by [3]

$$
Z_{N_{f}=2}(\hat{m})=I_{0}^{2}(\hat{m})-I_{1}^{2}(\hat{m}) .
$$

The $\mu$-dependence of the phase factor thus follows from the $\mu$-dependence of the phase quenched partition function (12)

$$
\left\langle e^{2 i \theta}\right\rangle_{1+1^{*}}=\frac{Z_{N_{f}=2}(\hat{m})}{Z_{1+1^{*}}(\hat{m} ; \hat{\mu})}=\frac{I_{0}^{2}(\hat{m})-I_{1}^{2}(\hat{m})}{2 e^{2 \hat{\mu}^{2}} \int_{0}^{1} d t t e^{-2 \hat{\mu}^{2} t^{2}} I_{0}^{2}(\hat{m} t)} .
$$

We now take the thermodynamic limit, $\hat{m} \rightarrow \infty$ and $\hat{\mu}^{2} \rightarrow \infty$, of the microscopic result for the phase factor in the phase quenched theory. In this limit the phase quenched theory has a second order phase transition at $\hat{m} /\left(2 \hat{\mu}^{2}\right)=1$. To show this we calculate the integral in (12) by a saddle point approximation. The transition between the two phases takes place when the saddle point hits the boundary of the integration region, i.e. when

$$
\bar{t}=\frac{\hat{m}}{2 \hat{\mu}^{2}}=1 .
$$

If we use the Gell-Mann-Oakes-Renner relation we find that this corresponds to the expected critical value of the chemical potential of $\mu=m_{\pi} / 2$.

For $\mu<m_{\pi} / 2$, the saddle point is outside the integration region and the leading contribution to the integral comes from the edge of the integral at $t=1$. In the thermodynamic limit we obtain (this is derived under the assumption that the integral is cut off by the exponential factor rather than the Gaussian factor, which in the thermodynamic limit is violated only very close to the critical point)

$$
Z_{1+1^{*}}(\hat{m} ; \hat{\mu}) \sim \frac{1}{2 \pi \hat{m}\left(\hat{m}-2 \hat{\mu}^{2}\right)} e^{2 \hat{m}} \quad \text { for } \quad \hat{m}>2 \hat{\mu}^{2} .
$$

We find that for $\hat{m}>2 \hat{\mu}^{2}$ the free energy of the phase quenched theory is $\mu$ independent in the thermodynamic limit. The thermodynamic limit of the microscopic two flavor partition function is

$$
Z_{N_{f}=2}(\hat{m} ; \hat{\mu}) \sim \frac{1}{2 \pi \hat{m}^{2}} e^{2 \hat{m}} .
$$

In the average phase (15) the free energies cancel. The $\mu$-dependence only enters through $1 / V$ corrections, i.e. through the pre-exponential factors

$$
\left\langle e^{2 i \theta}\right\rangle_{1+1^{*}} \sim\left(1-\frac{2 \hat{\mu}^{2}}{\hat{m}}\right) e^{0}=\left(1-\frac{4 \mu^{2}}{m_{\pi}^{2}}\right) e^{0}
$$


where we made use of the Gell-Mann-Oakes-Renner relation. This is precisely what we find from the general argument given in previous section. To see this we evaluate the two partition functions using (7). First, note that the particle content of the phase quenched partition function is a charged Goldstone boson and its anti-particle and two neutral Goldstone bosons. For $\mu<m_{\pi} / 2$, their masses are given by $m_{\pi}-2 \mu, m_{\pi}+2 \mu, m_{\pi}, m_{\pi}$, respectively, and the free energy is equal to by $2 m \Sigma$. The Jacobian in the integration measure is a constant. The ordinary two flavor partition function is of course obtained by setting $\mu=0$ in the expression obtained for the phase quenched partition function.

For $\mu>m_{\pi} / 2$ the thermodynamic limit of (12) allows us to determine both the free energy and the exponential prefactor. In this case the saddle point is inside the integration region and the saddle point approximation to the partition function (12) is given by

$$
Z_{1+1^{*}}(\hat{m} ; \hat{\mu}) \sim \sqrt{\frac{1}{2 \pi}} \frac{1}{\hat{\mu} \hat{m}} e^{\hat{m}^{2} / 2 \hat{\mu}^{2}+2 \hat{\mu}^{2}} \quad \text { for } \quad \hat{m}<2 \hat{\mu}^{2} .
$$

Notice that the prefactor of the exponential is larger by a factor of $\sqrt{V}$ than for $\mu<m_{\pi} / 2$. The reason is that the Goldstone mode with mass $m_{\pi}-2 \mu$ becomes exactly massless for $2 \mu>m_{\pi}$. To use the saddle point approximation one must introduce a pion condensate source term which lifts this mass away from zero (see Appendix A).

The two flavor partition function remains $\mu$ independent so that in the thermodynamic limit the average phase factor is given by

$$
\left\langle e^{2 i \theta}\right\rangle_{1+1^{*}} \sim\left\{\begin{array}{cc}
\left(1-\frac{4 \mu^{2}}{m_{\pi}^{2}}\right) e^{0} & \text { for } m_{\pi}>2 \mu, \\
\frac{1}{\sqrt{2 \pi V F_{\pi}^{4}}} \frac{2 \mu F_{\pi}}{m_{\pi}^{2}} e^{-2 V F_{\pi}^{2} \mu^{2}\left(1-m_{\pi}^{2} / 4 \mu^{2}\right)^{2}} & \text { for } m_{\pi}<2 \mu
\end{array}\right.
$$

Below we will derive the exact microscopic expression for the average phase factor in the quenched and unquenched theories. To perform the calculations we will make use of recently developed random matrix techniques.

\section{THE RANDOM MATRIX MODEL}

In order to compute the average phase factor in the microscopic limit we use a random matrix approach. In this limit the mass and chemical potential dependence of the QCD partition function is given by a random matrix partition function (see [39] for a review). The expectation value of the phase factor is then given by the ratio of two partition functions

$$
\left\langle e^{2 i \theta}\right\rangle_{N_{f}}=\frac{Z_{N_{f}+1 \mid 1^{*}}(m ; \mu)}{Z_{N_{f}}(m ; \mu)},
$$

where, in general, the random matrix partition function with $N_{f}$ quark flavors of mass $m_{f}$ and one pair of a regular quark and a conjugate bosonic quark both with mass $m$ is defined by

$$
Z_{N_{f}+1 \mid 1^{*}}\left(\left\{m_{f}\right\}, m ; \mu\right) \equiv \int d \Phi d \Psi w_{G}(\Phi) w_{G}(\Psi) \prod_{f=1}^{N_{f}} \operatorname{det}\left(\mathcal{D}(\mu)+m_{f}\right) \frac{\operatorname{det}(\mathcal{D}(\mu)+m)}{\operatorname{det}\left(\mathcal{D}^{\dagger}(\mu)+m\right)}
$$

In the computation of the average phase factor we always take the quark masses to be degenerate. The Dirac operator is given by

$$
\mathcal{D}(\mu)=\left(\begin{array}{cc}
0 & i \Phi+\mu \Psi \\
i \Phi^{\dagger}+\mu \Psi^{\dagger} & 0
\end{array}\right)
$$

Here, $\Phi$ and $\Psi$ are complex $N \times N$ matrices with the same Gaussian weight function

$$
w_{G}(X)=\exp \left(-N \operatorname{Tr} X^{\dagger} \mathrm{X}\right) .
$$

The partition function $Z_{N_{f}}(m ; \mu)$ with $N_{f}$ flavors of mass $m$ is given by (23) without the ratio of the two determinants multiplying the $N_{f}$ flavor quark determinant. The microscopic limit of the random matrix model is obtained by taking the size of the matrices infinitely large, $N \rightarrow \infty$, while keeping the products

$$
\hat{m}=2 m N \quad \text { and } \quad \hat{\mu}^{2}=2 \mu^{2} N
$$


fixed. Expressions involving the microscopic partition function are denoted by explicitly writing the microscopic variables $\hat{\mu}$ and $\hat{m}$.

The random matrix partition function (23) can be rewritten in terms of an integral over the eigenvalues of $\mathcal{D}$ and the unitary matrices that diagonalize $\mathcal{D}$. The integral over the unitary matrices can be performed analytically [32] resulting in the eigenvalue representation of the partition function

$$
Z_{N_{f}+1 \mid 1^{*}}\left(\left\{m_{f}\right\}, m ; \mu\right) \sim \int_{\mathbb{C}} \prod_{k=1}^{N} d^{2} z_{k} \mathcal{P}_{N_{f}+1 \mid 1^{*}}\left(\left\{z_{i}\right\},\left\{z_{i}^{*}\right\} ; \mu\right),
$$

where the integration extends over the full complex plane. The joint probability distribution of the eigenvalues is given by

$$
\mathcal{P}_{N_{f}+1 \mid 1^{*}}\left(\left\{z_{i}\right\},\left\{z_{i}^{*}\right\} ; \mu\right)=\frac{1}{\mu^{2 N}}\left|\Delta_{N}\left(\left\{z_{l}^{2}\right\}\right)\right|^{2} \prod_{k=1}^{N} w\left(z_{k}, z_{k}^{*} ; \mu\right) \prod_{f=1}^{N_{f}}\left(m_{f}^{2}-z_{k}^{2}\right) \frac{\left(m^{2}-z_{k}^{2}\right)}{\left(m^{2}-z_{k}^{* 2}\right)} .
$$

The Vandermonde determinant is defined as

$$
\Delta_{N}\left(\left\{z_{l}^{2}\right\}\right) \equiv \prod_{i>j=1}^{N}\left(z_{i}^{2}-z_{j}^{2}\right)
$$

and the weight function reads 32 ]

$$
w\left(z_{k}, z_{k}^{*} ; \mu\right)=\left|z_{k}\right|^{2} K_{0}\left(\frac{N\left(1+\mu^{2}\right)}{2 \mu^{2}}\left|z_{k}\right|^{2}\right) \exp \left(-\frac{N\left(1-\mu^{2}\right)}{4 \mu^{2}}\left(z_{k}^{2}+z_{k}^{* 2}\right)\right) .
$$

The eigenvalue representation makes it possible to define orthogonal polynomials in the complex plane 32, 40, 41, 42, 43, 44, 45]. In order to evaluate the average phase factor we will make use of such orthogonal polynomials and their Cauchy transform. The complex Laguerre polynomials given by [32]

$$
p_{k}(z ; \mu)=\left(\frac{1-\mu^{2}}{N}\right)^{k} k ! L_{k}\left(-\frac{N z^{2}}{1-\mu^{2}}\right)
$$

are the orthogonal polynomials corresponding to the weight $w\left(z, z^{*} ; \mu\right)$ given in (30). They satisfy the orthogonality relation

$$
\int_{\mathbb{C}} d^{2} z w\left(z, z^{*} ; \mu\right) p_{k}(z ; \mu) p_{l}(z ; \mu)^{*}=r_{k} \delta_{k l}
$$

with the norm

$$
r_{k}=\frac{\pi \mu^{2}\left(1+\mu^{2}\right)^{2 k} k ! k !}{N^{2 k+2}} .
$$

The Cauchy transform of the orthogonal polynomials is defined as

$$
h_{k}(m ; \mu)=\int_{\mathbb{C}} d^{2} z \frac{1}{z^{2}-m^{2}} w\left(z, z^{*} ; \mu\right) p_{k}^{*}(z ; \mu),
$$

where $\mathbb{C}$ indicates that the integration extends over the complex plane.

\section{THE AVERAGE PHASE FACTOR IN THE MICROSCOPIC LIMIT}

Partition functions that are given by expectation values of ratios of determinants can be expressed simply in terms of orthogonal polynomials and their Cauchy transforms [31, 40, 41, 42, 43, 44, 45]. Generalizing the results of [45] we find that the partition function (23) for matrix size $N$ is given by

$$
\mathcal{Z}_{N_{f}+1 \mid 1^{*}}\left(\left\{m_{f}\right\}, m ; \mu\right)=\frac{1}{r_{N-1} \Delta_{N_{f}+1}\left(\left\{m_{f}^{2}\right\}\right)}\left|\begin{array}{lll}
h_{N-1}(m ; \mu) & \cdots & h_{N+N_{f}}(m ; \mu) \\
p_{N-1}(m) & \cdots & p_{N+N_{f}}(m) \\
p_{N-1}\left(m_{1}\right) & \cdots & p_{N+N_{f}}\left(m_{1}\right) \\
\vdots & & \vdots \\
p_{N-1}\left(m_{N_{f}}\right) & \cdots & p_{N+N_{f}}\left(m_{N_{f}}\right)
\end{array}\right|
$$


In order to make contact with QCD we take the microscopic limit of this result. The orthogonal polynomials corresponding to the weight (30) are the complex Laguerre polynomials. In the microscopic limit, $\hat{z}=2 N z$ for $N \rightarrow \infty$, the polynomial $p_{N-1} / r_{N-1}$ is

$$
\frac{p_{N-1}(\hat{z} ; \hat{\mu})}{r_{N-1}} \sim e^{-2 \hat{\mu}^{2}} I_{0}(\hat{z})
$$

where we have adopted the normalization used in [47]. Taking the limit of all quark masses equal to $m$ we obtain in the microscopic limit (also in the remainder of the paper the quark masses will be taken equal to $m$, which is indicated by the notation)

$$
Z_{N_{f}+1 \mid 1^{*}}(\hat{m} ; \hat{\mu})=\frac{1}{2^{N_{f}+1}} \frac{1}{\hat{m}^{N_{f}\left(N_{f}+1\right)}}\left|\begin{array}{lll}
X^{(0)}(\hat{m} ; \hat{\mu}) & \cdots & X^{\left(N_{f}+1\right)}(\hat{m} ; \hat{\mu}) \\
I_{0}(\hat{m}) & \cdots & \delta_{\hat{m}}^{N_{f}+1} I_{0}(\hat{m}) \\
\vdots & & \vdots \\
\delta_{\hat{m}}^{N_{f}} I_{0}(\hat{m}) & \cdots & \delta_{\hat{m}}^{2 N_{f}+1} I_{0}(\hat{m})
\end{array}\right| .
$$

Here, $\delta_{\hat{m}} \equiv \hat{m} \frac{d}{d \hat{m}}$ and

$$
X^{(k)}(\hat{m} ; \hat{\mu}) \equiv-\frac{1}{4 \pi} \frac{1}{\hat{\mu}^{2}} e^{-2 \hat{\mu}^{2}} \int_{\mathbb{C}} \mathrm{d}^{2} z \frac{w\left(z, z^{*}, \hat{\mu}\right) \delta_{z^{*}}^{k} I_{0}\left(z^{*}\right)}{z^{2}-\hat{m}^{2}} .
$$

This result was first presented in the letter [37]. On the next pages we examine this result in detail.

Denoting the integrand of the $X^{(k)}$ by $F^{(k)}\left(z, z^{*}\right)$, we conclude from the properties of Bessel functions that $\left[F^{(k)}\left(z, z^{*}\right)\right]^{*}=F^{(k)}\left(z^{*}, z\right)$. Therefore, the imaginary part of the $X^{(k)}$ vanishes after integration over $d^{2} z$.

By using the identity $(z=x+i y)$

$$
I_{k}\left(z^{*}\right)=\frac{(-1)^{k}}{\pi} \frac{\sqrt{z^{*}}}{\sqrt{-z^{*}}}\left(K_{k}\left(z^{*}\right)-(-1)^{k} K_{k}\left(-z^{*}\right)\right), \quad k=0,1,2, \cdots
$$

we can perform the $y$-integral in (38) by a contour integral in the complex $y$-plane. By changing variables $z \rightarrow-z$ and $z^{*} \rightarrow-z^{*}$ it can be easily shown that both terms in (39) give the same integral. Our result is thus given by

$$
X^{(k)}(\hat{m} ; \hat{\mu})=\frac{1}{2 \pi^{2}} \frac{1}{\hat{\mu}^{2}} e^{-2 \hat{\mu}^{2}} \int_{-\infty}^{\infty} d x \int_{-\infty}^{\infty} d y \frac{|z|^{2}}{z^{2}-\hat{m}^{2}} K_{0}\left(\frac{|z|^{2}}{4 \hat{\mu}^{2}}\right) \exp \left(-\frac{\left(z^{2}+z^{* 2}\right)}{8 \hat{\mu}^{2}}\right) \delta_{z^{*}}^{k}\left[\frac{\sqrt{z^{*}}}{\sqrt{-z^{*}}} K_{0}\left(-z^{*}\right)\right] .
$$

The only nonanalyticities in the complex $y$-plane are on the imaginary $y$-axis, and for $|y| \rightarrow \infty$, the integrand vanishes sufficiently fast in the lower part of the complex $y$-plane to deform the integration contour as

$$
\begin{aligned}
& \int_{-\infty}^{-\epsilon} d y \cdots \rightarrow-\int_{-\epsilon}^{-\epsilon-i \infty} d y \cdots \\
& \int_{\epsilon}^{\infty} d y \cdots \rightarrow \int_{\epsilon}^{\epsilon-i \infty} d y \cdots
\end{aligned}
$$

We integrate over the new contour by parameterizing $y=-i s \pm \epsilon$ which gives an additional $-i$ from the Jacobian. The pole term can be decomposed into a principal value part and a $\delta$-function,

$$
\begin{aligned}
\frac{1}{z^{2}-\hat{m}^{2}} & =\frac{1}{2 \hat{m}}\left(\frac{1}{z-\hat{m}}-\frac{1}{z+\hat{m}}\right) \\
& =\frac{1}{2 \hat{m}}\left[\frac{x+s-\hat{m} \mp i \epsilon}{(x+s-\hat{m})^{2}+\epsilon^{2}}-\frac{x+s+\hat{m} \mp i \epsilon}{(x+s+\hat{m})^{2}+\epsilon^{2}}\right] .
\end{aligned}
$$

The principal value part combines with the discontinuity accross the negative imaginary $y$-axis of the other factors in the integrand in (40),

$$
\begin{aligned}
X_{\text {cut }}^{(k)}(\hat{m} ; \hat{\mu})= & \frac{i}{2 \pi^{2}} \frac{1}{\hat{\mu}^{2}} e^{-\frac{\hat{m}^{2}}{8 \hat{\mu}^{2}}} e^{-2 \hat{\mu}^{2}} \int_{0}^{\infty} d s \int_{-\infty}^{\infty} d x|z|^{2} \frac{1}{2 \hat{m}}\left[\mathcal{P} \frac{1}{x+s+\hat{m}}-\mathcal{P} \frac{1}{x+s-\hat{m}}\right] \\
& \times \operatorname{Disc}\left[K_{0}\left(\frac{|z|^{2}}{4 \hat{\mu}^{2}}\right) \exp \left(-\frac{\left(z^{2}+z^{* 2}\right)}{8 \hat{\mu}^{2}}\right) \delta_{z^{*}}^{k}\left[\frac{\sqrt{z^{*}}}{\sqrt{-z^{*}}} K_{0}\left(-z^{*}\right)\right]\right]
\end{aligned}
$$


Using the orthogonality of the polynomials on which this integral is based, it can be shown that this integral vanishes [46]

$$
X_{\text {cut }}^{(k)}(\hat{m} ; \hat{\mu})=0 .
$$

What remains is the $\delta$-function part of (42) in combination with the part of the of factors in the integrand that is continuous accross the negative imaginary $y$-axis. By inspection one easily finds that the contribution of the pole at $s=-x-\hat{m}$ vanishes. For the contribution of the pole at $s=x-\hat{m}$ we have to distinguish $\hat{m}-x<x$ and $\hat{m}-x>x$. Because $\sqrt{z^{*}} / \sqrt{-z^{*}}$ has a cut for all values of $s$ (notice that $z^{*}=x-s \mp \epsilon$ ), in the first case the nonvanishing contribution comes from the cut in $K_{0}\left(|z|^{2}\right)$ and in the second case from the cut in $K_{0}\left(-z^{*}\right)$. We thus find

$$
\begin{aligned}
X^{(k)}(\hat{m} ; \hat{\mu})= & -\frac{1}{4 \hat{\mu}^{2}} e^{-\frac{\hat{m}^{2}}{8 \hat{\mu}^{2}}} e^{-2 \hat{\mu}^{2}}\left[\int_{-\infty}^{\hat{m} / 2} d x(2 x-\hat{m}) I_{0}\left(\frac{(2 x-\hat{m}) \hat{m}}{4 \hat{\mu}^{2}}\right) \exp \left(-\frac{(\hat{m}-2 x)^{2}}{8 \hat{\mu}^{2}}\right) \delta_{\hat{m}-2 x}^{k}\left[K_{0}(\hat{m}-2 x)\right]\right. \\
& \left.-\int_{\hat{m} / 2}^{\hat{m}} d x(2 x-\hat{m}) K_{0}\left(\frac{(2 x-\hat{m}) \hat{m}}{4 \hat{\mu}^{2}}\right) \exp \left(-\frac{(2 x-\hat{m})^{2}}{8 \hat{\mu}^{2}}\right) \delta_{2 x-\hat{m}}^{k}\left[I_{0}(2 x-\hat{m})\right]\right] .
\end{aligned}
$$

This result can be simplified to

$$
\begin{aligned}
X^{(k)}(\hat{m} ; \hat{\mu})= & e^{-2 \hat{\mu}^{2}} \frac{1}{4 \hat{\mu}^{2}} e^{-\frac{\hat{m}^{2}}{8 \hat{\mu}^{2}}} \\
& \times\left[\int_{0}^{\hat{m}} d u u \exp \left[-\frac{u^{2}}{8 \hat{\mu}^{2}}\right] K_{0}\left(\frac{u \hat{m}}{4 \hat{\mu}^{2}}\right)\left(u \partial_{u}\right)^{k} I_{0}(u)+\int_{0}^{\infty} d u u \exp \left[-\frac{u^{2}}{8 \hat{\mu}^{2}}\right] I_{0}\left(\frac{u \hat{m}}{4 \hat{\mu}^{2}}\right)\left(u \partial_{u}\right)^{k} K_{0}(u)\right] .
\end{aligned}
$$

Making use of the identities derived in Appendix B these integrals can be rewritten as the sum of a polynomial in $\mu^{2}$ and an integral with an essential singularity at $\mu=0$,

$$
\begin{aligned}
X^{(0)}(\hat{m} ; \hat{\mu})= & K_{0}(\hat{m})-e^{-2 \hat{\mu}^{2}} \frac{1}{4 \hat{\mu}^{2}} e^{-\frac{\hat{m}^{2}}{8 \hat{\mu}^{2}}} \int_{\hat{m}}^{\infty} d u u \exp \left[-\frac{u^{2}}{8 \hat{\mu}^{2}}\right] K_{0}\left(\frac{u \hat{m}}{4 \hat{\mu}^{2}}\right) I_{0}(u), \\
X^{(1)}(\hat{m} ; \hat{\mu})= & -\hat{m} K_{1}(\hat{m})+4 \hat{\mu}^{2} K_{0}(\hat{m})-e^{-2 \hat{\mu}^{2}} \frac{1}{4 \hat{\mu}^{2}} e^{-\frac{\hat{m}^{2}}{8 \hat{\mu}^{2}}} \int_{\hat{m}}^{\infty} d u u \exp \left[-\frac{u^{2}}{8 \hat{\mu}^{2}}\right] K_{0}\left(\frac{u \hat{m}}{4 \hat{\mu}^{2}}\right) u \partial_{u} I_{0}(u), \\
X^{(2)}(\hat{m} ; \hat{\mu})= & K_{0}(\hat{m})\left(\hat{m}^{2}+16 \hat{\mu}^{4}+8 \hat{\mu}^{2}\right)-8 K_{1}(\hat{m}) \hat{m} \hat{\mu}^{2} \\
& -e^{-2 \hat{\mu}^{2}} \frac{1}{4 \hat{\mu}^{2}} e^{-\frac{\hat{m}^{2}}{8 \hat{\mu}^{2}}} \int_{\hat{m}}^{\infty} d u u \exp \left[-\frac{u^{2}}{8 \hat{\mu}^{2}}\right] K_{0}\left(\frac{u \hat{m}}{4 \hat{\mu}^{2}}\right)\left(u \partial_{u}\right)^{2} I_{0}(u), \\
X^{(3)}(\hat{m} ; \hat{\mu})= & K_{0}(\hat{m})\left(12 \hat{m}^{2} \hat{\mu}^{2}+64 \hat{\mu}^{6}+96 \hat{\mu}^{4}+2 \hat{m}^{2}+16 \hat{\mu}^{2}\right)-K_{1}(\hat{m})\left(48 \hat{m} \hat{\mu}^{4}+\hat{m}^{3}+24 \hat{m} \hat{\mu}^{2}\right) \\
& -e^{-2 \hat{\mu}^{2}} \frac{1}{4 \hat{\mu}^{2}} e^{-\frac{\hat{m}^{2}}{8 \hat{\mu}^{2}}} \int_{\hat{m}}^{\infty} d u u \exp \left[-\frac{u^{2}}{8 \hat{\mu}^{2}}\right] K_{0}\left(\frac{u \hat{m}}{4 \hat{\mu}^{2}}\right)\left(u \partial_{u}\right)^{3} I_{0}(u),
\end{aligned}
$$

The analytic properties of the $X^{(k)}$ will be discussed further in section VII.

In oder to complete the computation of the average phase factor (22) we only need to recall that the ordinary $(\mu$ independent) microscopic partition function is given by [3]

$$
Z_{N_{f}}(\hat{m}) \sim \hat{m}^{-N_{f}\left(N_{f}-1\right)} \operatorname{det}\left[\delta_{\hat{m}}^{k+l} I_{0}(\hat{m})\right]_{k, l=0, \ldots, N_{f}-1} .
$$

In the next section we will discuss explicitly the result for the average phase factor for $N_{f}=0,1$ and 2 .

\section{EXPLICIT RESULTS AND THE THERMODYNAMIC LIMIT OF THE PHASE FACTOR}

In this section we will take a closer look at several specific cases and derive the large $\hat{m}$ and large $\hat{\mu}$ asymptotic expansions of the average phase factor from the exact microscopic expressions given in the previous section. We refer to this as the thermodynamic limit of the microscopic results. These results confirm the expressions for the average phase factor obtained in section $\amalg$ from the mean field argument. For the technical details of the asymptotic expansion we refer to Appendix $\mathrm{C}$. 


\section{The Quenched Case}

We first consider the expectation value of the phase factor in the quenched case. Then

$$
\begin{aligned}
\left\langle e^{2 i \theta}\right\rangle_{N_{f}=0}= & \left|\begin{array}{cc}
X^{(0)}(\hat{m} ; \hat{\mu}) & X^{(1)}(\hat{m} ; \hat{\mu}) \\
I_{0}(\hat{m}) & \hat{m} I_{1}(\hat{m})
\end{array}\right| \\
= & 1-4 \hat{\mu}^{2} I_{0}(\hat{m}) K_{0}(\hat{m}) \\
& -e^{-2 \hat{\mu}^{2}} \frac{1}{4 \hat{\mu}^{2}} e^{-\frac{\hat{m}^{2}}{8 \hat{\mu}^{2}}} \int_{\hat{m}}^{\infty} d x x \exp \left[-\frac{x^{2}}{8 \hat{\mu}^{2}}\right] K_{0}\left(\frac{x \hat{m}}{4 \hat{\mu}^{2}}\right)\left(I_{0}(x) \hat{m} I_{1}(\hat{m})-x I_{1}(x) I_{0}(\hat{m})\right),
\end{aligned}
$$

where we made use of the Wronskian identity $\hat{m} I_{0}(\hat{m}) K_{1}(\hat{m})+\hat{m} I_{1}(\hat{m}) K_{0}(\hat{m})=1$.

We now take the thermodynamic limit $\hat{m} \rightarrow \infty$ and $\hat{\mu} \rightarrow \infty$. In the normal phase we can use the leading order asymptotic expansions for the $X^{(k)}$ and the Bessel functions whereas in the condensed phase we have to include the subleading corrections. Substituting the expressions obtained in Appendix $\mathbb{C}$ we find (the result for $m_{\pi}<2 \mu$ is not valid very close to the critical point (see Appendix C))

$$
\left\langle e^{2 i \theta}\right\rangle_{N_{f}=0} \sim\left\{\begin{array}{cc}
\left(1-\frac{4 \mu^{2}}{m_{\pi}^{2}}\right) e^{0} & \text { for } m_{\pi}>2 \mu, \\
\frac{1}{V^{3 / 2} \sqrt{2 \pi}} \frac{1}{m_{\pi}^{2} F_{\pi}^{3} \mu} \frac{1}{2\left(1-m_{\pi}^{2} / 4 \mu^{2}\right)^{2}} e^{-2 V F_{\pi}^{2} \mu^{2}\left(1-m_{\pi}^{2} / 4 \mu^{2}\right)^{2}} & \text { for } m_{\pi}<2 \mu .
\end{array}\right.
$$

For $m_{\pi}<2 \mu$ the leading order terms contributing to the prefactor cancel. This asymptotic result agrees with the result we have obtained from the general arguments in section [I]

\section{Full $Q C D$ for $N_{f}=1$}

For one flavor, the explicit microscopic expression for the expectation value of the phase factor is given by

$$
\left\langle e^{2 i \theta}\right\rangle_{N_{f}=1}=\frac{1}{2 \hat{m}^{2} I_{0}(\hat{m})}\left|\begin{array}{lll}
X^{(0)}\left(\hat{m}^{*} ; \hat{\mu}\right) & X^{(1)}\left(\hat{m}^{*} ; \hat{\mu}\right) & X^{(2)}\left(\hat{m}^{*} ; \hat{\mu}\right) \\
I_{0}(\hat{m}) & \hat{m} I_{1}(\hat{m}) & \hat{m}^{2} I_{0}(\hat{m}) \\
\hat{m} I_{1}(\hat{m}) & \hat{m}^{2} I_{0}(\hat{m}) & \hat{m}^{2}\left(2 I_{0}(\hat{m})+\hat{m} I_{1}(\hat{m})\right)
\end{array}\right|,
$$

where the $X^{(k)}$ have been given in the previous section and we used that $Z_{N_{f}=1}(\hat{m} ; \hat{\mu})=I_{0}(\hat{m})$. With the expressions found for $X^{(k)}$ the average phase factor can be written as

$$
\begin{aligned}
& \left\langle e^{2 i \theta}\right\rangle_{N_{f}=1}=1-4\left(\hat{m} K_{0}(\hat{m}) I_{0}(\hat{m})+\frac{I_{1}(\hat{m})^{2}}{I_{0}(\hat{m})}\left(K_{0}(\hat{m})-\hat{m} K_{1}(\hat{m})\right)\right) \hat{\mu}^{2}+8 K_{0}(\hat{m}) \frac{I_{0}(\hat{m})^{2}-I_{1}(\hat{m})^{2}}{I_{0}(\hat{m})} \hat{\mu}^{4} \\
& -\frac{e^{-2 \hat{\mu}^{2}-\frac{\hat{m}^{2}}{8 \hat{\mu}^{2}}}}{8 \hat{m}^{2} \hat{\mu}^{2} I_{0}(\hat{m})} \int_{\hat{m}}^{\infty} d x x \exp \left[-\frac{x^{2}}{8 \hat{\mu}^{2}}\right] K_{0}\left(\frac{x \hat{m}}{4 \hat{\mu}^{2}}\right)\left|\begin{array}{lll}
I_{0}(x) & \delta_{x} I_{1}(x) & \left(\delta_{x}\right)^{2} I_{0}(x) \\
I_{0}(\hat{m}) & \delta_{\hat{m}} I_{0}(\hat{m}) & \left(\delta_{\hat{m}}\right)^{2} I_{0}(\hat{m}) \\
\delta_{\hat{m}} I_{0}(\hat{m}) & \left(\delta_{\hat{m}}\right)^{2} I_{0}(\hat{m}) & \left(\delta_{\hat{m}}\right)^{3} I_{0}(\hat{m})
\end{array}\right| .
\end{aligned}
$$

The large $\hat{m}$ and $\hat{\mu}$ limit of the expectation value of the phase follows from the asymptotic expressions for the $X^{(k)}$ given in Appendix $\mathbb{C}$ and the asymptotic expressions for the Bessel functions

$$
\left\langle e^{2 i \theta}\right\rangle_{N_{f}=1} \sim\left\{\begin{array}{cc}
\left(1-\frac{4 \mu^{2}}{m_{\pi}^{2}}\right)^{2} e^{0} & \text { for } m_{\pi}>2 \mu \\
O\left(\frac{1}{V^{5 / 2}}\right) e^{-2 V F_{\pi}^{2} \mu^{2}\left(1-m_{\pi}^{2} / 4 \mu^{2}\right)^{2}} & \text { for } m_{\pi}<2 \mu
\end{array}\right.
$$

The first three orders contributing to the prefactor of the result for $m_{\pi}<2 \mu$ vanish.

Since the average phase factor is the ratio of two partition functions it is necessarily real even if the "statistical weight" is complex such as for $N_{f}=1$. This does not imply that $\langle\sin 2 \theta\rangle$ vanishes such as in the quenched theory or the phase quenched theory. Since $\langle\sin 2 \theta\rangle=\langle\exp (2 i \theta)-\exp (-2 i \theta)\rangle / 2 i$ and

$$
\left\langle e^{-2 i \theta}\right\rangle_{N_{f}=1}=\frac{Z_{N_{f}=1^{*}}(m ; \mu)}{Z_{N_{f}=1}(m ; \mu)}=1,
$$


we have in the microscopic limit that

$$
\langle\sin 2 \theta\rangle_{N_{f}=1}=\frac{1}{2 i}\left[\left\langle e^{2 i \theta}\right\rangle_{N_{f}=1}-1\right] .
$$

We thus find that the expectation value $\langle\sin 2 \theta\rangle_{N_{f}=1}$ is generally nonzero and purely imaginary. Its thermodynamic limit is given by

$$
\langle\sin (2 \theta)\rangle_{N_{f}=1} \sim\left\{\begin{array}{cc}
\frac{1}{2 i}\left(\left(1-\frac{4 \mu^{2}}{m_{\pi}^{2}}\right)^{2}-1\right) & \text { for } m_{\pi}>2 \mu, \\
-\frac{1}{2 i} & \text { for } m_{\pi}<2 \mu .
\end{array}\right.
$$

Since the average of the inverse phase factor is 1 we automatically also know the variance of the phase factor

$$
\left\langle e^{2 i \theta} e^{-2 i \theta}\right\rangle_{N_{f}=1}-\left\langle e^{2 i \theta}\right\rangle_{N_{f}=1}\left\langle e^{-2 i \theta}\right\rangle_{N_{f}=1}=1-\left\langle e^{2 i \theta}\right\rangle_{N_{f}=1}
$$

So while the average phase factor goes to zero, its variance goes to 1 for $\mu>m_{\pi} / 2$, what would also have been the result for a uniformly random distribution of the phase. This central feature of the sign problem is also present for larger number of flavors as well as for quenched and phase quenched QCD (in the latter two cases there is of course no sign problem).

\section{Two dynamical flavors}

With two flavors the explicit microscopic expression for the average phase factor is

$$
\begin{aligned}
\left\langle e^{2 i \theta}\right\rangle_{N_{f}=2} & =\frac{1}{8 \hat{m}^{6}\left(I_{0}(\hat{m})^{2}-I_{1}(\hat{m})^{2}\right)} \\
& \times\left|\begin{array}{llll}
X^{(0)}\left(\hat{m}^{*} ; \hat{\mu}\right) & X^{(1)}\left(\hat{m}^{*} ; \hat{\mu}\right) & X^{(2)}\left(\hat{m}^{*} ; \hat{\mu}\right) & X^{(3)}\left(\hat{m}^{*} ; \hat{\mu}\right) \\
I_{0}(\hat{m}) & \delta_{\hat{m}} I_{0}(\hat{m}) & \left(\delta_{\hat{m}}\right)^{2} I_{0}(\hat{m}) & \left(\delta_{\hat{m}}\right)^{3} I_{0}(\hat{m}) \\
\delta_{\hat{m}} I_{0}(\hat{m}) & \left(\delta_{\hat{m}}\right)^{2} I_{0}(\hat{m}) & \left(\delta_{\hat{m}}\right)^{3} I_{0}(\hat{m}) & \left(\delta_{\hat{m}}\right)^{4} I_{0}(\hat{m}) \\
\left(\delta_{\hat{m}}\right)^{2} I_{0}(\hat{m}) & \left(\delta_{\hat{m}}\right)^{3} I_{0}(\hat{m}) & \left(\delta_{\hat{m}}\right)^{4} I_{0}(\hat{m}) & \left(\delta_{\hat{m}}\right)^{5} I_{0}(\hat{m})
\end{array}\right| .
\end{aligned}
$$

For $\mu<m_{\pi} / 2$ the thermodynamic limit of this result is

$$
\left\langle e^{2 i \theta}\right\rangle_{N_{f}=2} \sim\left(1-\frac{4 \mu^{2}}{m_{\pi}^{2}}\right)^{3} e^{0} \quad \text { for } \quad m_{\pi}>2 \mu .
$$

As the chemical potential increases beyond half the pion mass the average phase factor is again exponentially small in the volume in agreement with (9). The fast convergence to the thermodynamic limit is illustrated in figure 1 .

\section{ANALYTIC CONTINUATION FROM IMAGINARY CHEMICAL POTENTIAL}

For purely imaginary chemical potential the fermion determinant is real, and the phase factor is equal to unity. Thus it seems to be impossible to calculate the average phase factor at imaginary values of $\mu$ and then perform an analytic continuation in $\mu$. However, if we first use that $\operatorname{det}\left(D+\mu \gamma_{0}+m\right)^{*}=\operatorname{det}\left(D-\mu \gamma_{0}+m\right)$ to write the average phase factor at real chemical potential as

$$
\left\langle e^{2 i \theta(\mu)}\right\rangle_{N_{f}}=\left\langle\frac{\operatorname{det}\left(D+\mu \gamma_{0}+m\right)}{\operatorname{det}\left(D-\mu \gamma_{0}+m\right)}\right\rangle_{N_{f}}
$$

and subsequently substitute $\mu$ by $i \mu$ we obtain a real number

$$
\left\langle e^{2 i \theta(i \mu)}\right\rangle_{N_{f}} \equiv\left\langle\frac{\operatorname{det}\left(D+i \mu \gamma_{0}+m\right)}{\operatorname{det}\left(D-i \mu \gamma_{0}+m\right)}\right\rangle_{N_{f}}
$$

that is (typically) different from one. Below we will use this analytical continuation between real and imaginary chemical potential. 

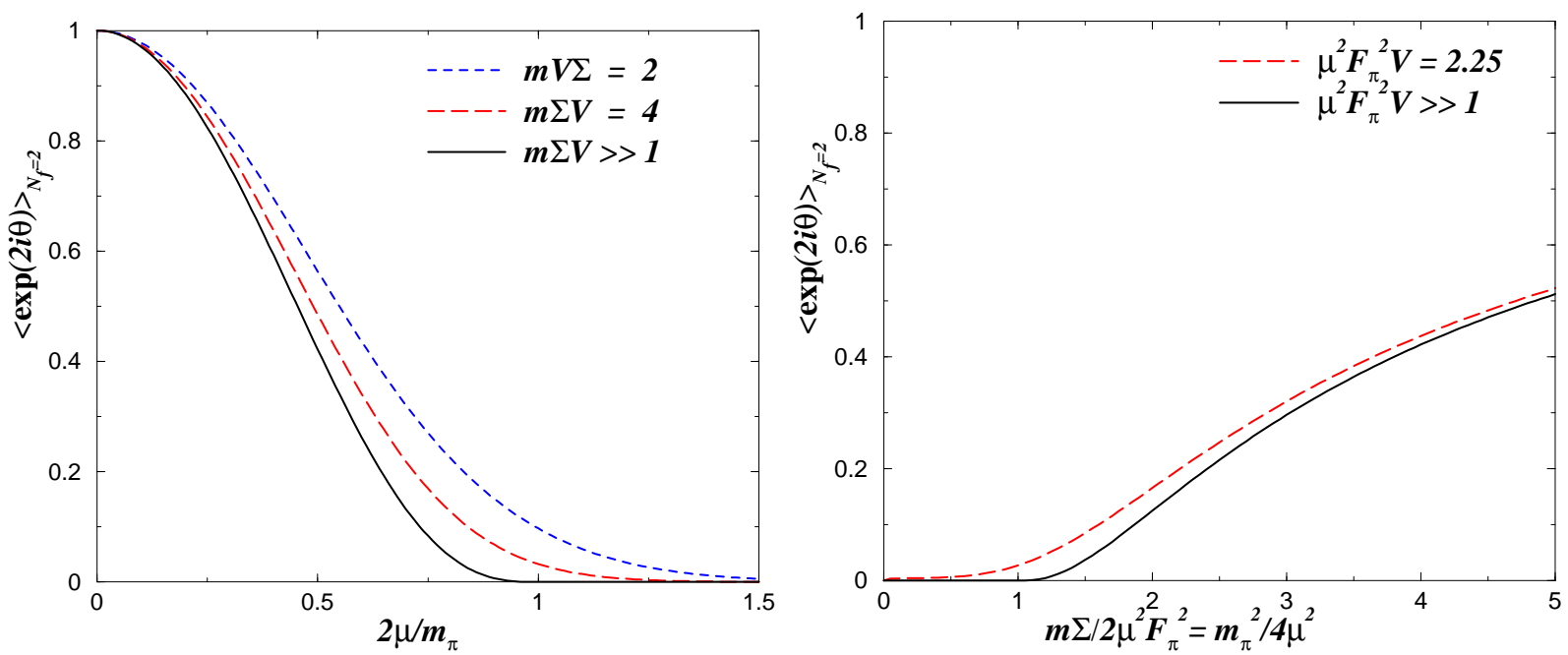

FIG. 1: Left: The average phase factor for two dynamical flavors as a function of the chemical potential for fixed quark mass. The full curve represents the result in the thermodynamic limit. Note that the convergence to the thermodynamic limit is particular rapid for small values of the chemical potential. Right: The average phase factor as a function of the quark mass for fixed chemical potential. The full curve again displays the result in the thermodynamic limit.

The microscopic limit of the quenched average of (65) for imaginary chemical potential can be derived by means of the supersymmetric method. It is given by [55]

$$
\left\langle e^{2 i \theta(i \mu)}\right\rangle_{N_{f}=0}=1+4 \hat{\mu}^{2} I_{0}(\hat{m}) K_{0}(\hat{m}) .
$$

Since the result is a polynomial in $\hat{\mu}^{2}$, analytical continuation to real chemical potential is trivial: we simply flip the sign of $\mu^{2}$. This result should be contrasted with the result for real $\mu$ given in Eq. (53):

$$
\begin{aligned}
\left\langle e^{2 i \theta(\mu)}\right\rangle_{N_{f}=0} & =1-4 \hat{\mu}^{2} I_{0}(\hat{m}) K_{0}(\hat{m})-\frac{e^{-2 \hat{\mu}^{2}}}{4 \hat{\mu}^{2}} e^{-\frac{\hat{m}^{2}}{8 \hat{\mu}^{2}}} \int_{\hat{m}}^{\infty} d x x e^{-\frac{x^{2}}{8 \hat{\mu}^{2}}} K_{0}\left(\frac{x \hat{m}}{4 \hat{\mu}^{2}}\right)\left(I_{0}(x) \hat{m} I_{1}(\hat{m})-x I_{1}(x) I_{0}(\hat{m})\right) \\
& \sim 1-4 \hat{\mu}^{2} I_{0}(\hat{m}) K_{0}(\hat{m})+\hat{\mu}^{2} K_{0}\left(\frac{\hat{m}^{2}}{4 \hat{\mu}^{2}}\right) e^{-\frac{\hat{m}^{2}}{4 \hat{\mu}^{2}}-2 \hat{\mu}^{2}}\left(I_{0}(\hat{m})^{2}-I_{1}(\hat{m})^{2}\right) \quad \text { for } \quad \hat{\mu} \rightarrow 0 .
\end{aligned}
$$

What is reproduced by the expression (66) for purely imaginary chemical potential are the analytic terms in $\mu$, but the term with the essential singularity is not obtained. Note that the non-analytic term does not contribute to the Taylor series of (67). The Taylor expansion around $\mu=0$ of (67) is thus given by the first two terms only. This expansion can be analytically continued in $\mu$ and necessarily reproduces the result for imaginary $\mu$ because its Taylor expansion is finite.

The microscopic limit of the average phase factor for imaginary $\mu$ can also be evaluated for $N_{f} \neq 0$ using the supersymmetric method of [48]. However, it is possible to obtain $\langle\exp (2 i \theta(i \mu))\rangle_{N_{f}}$ from the results at real $\mu$ without a detailed computation. We only need that the average phase factor for imaginary $\mu$ is given by a finite power series in $\mu^{2}$. This immediately follows from the super symmetric approach where $i \mu$ only occurs in combination with Grassmann variables. Therefore, also for $N_{f} \neq 0$, the exact average phase factor at imaginary $\mu$ can be obtained from the analytical continuation of the Taylor expansion of the expression for real $\mu$. For example, for $N_{f}=1$ we obtain the result

$$
\left\langle e^{2 i \theta(i \mu)}\right\rangle_{N_{f}=1}=1+4\left(\hat{m} K_{0}(\hat{m}) I_{0}(\hat{m})+\frac{I_{1}(\hat{m})^{2}}{I_{0}(\hat{m})}\left(K_{0}(\hat{m})-\hat{m} K_{1}(\hat{m})\right)\right) \hat{\mu}^{2}+8 K_{0}(\hat{m}) \frac{I_{0}(\hat{m})^{2}-I_{1}(\hat{m})^{2}}{I_{0}(\hat{m})} \hat{\mu}^{4} .
$$

Conversely, if we would have calculated the "phase factor" for imaginary chemical potential and then made the analytical continuation to real $\mu$ we only would have obtained the polynomial terms in $\mu$. Terms for which all derivatives at $\mu=0$ vanish cannot possibly be obtained from a Taylor expansion.

For $\mu \ll m_{\pi} / 2$ the term with the essential singularity is not important. However, it becomes the dominant term when $\mu$ approaches $m_{\pi} / 2$. For $\mu>m_{\pi} / 2$ the non-analytic term cancels the terms that are polynomial in $\mu$, and is responsible for the exponential suppression of average phase factor. In the thermodynamic limit, the non-analytic 
term has a phase transition at $\mu=m_{\pi} / 2$. For $\mu<m_{\pi} / 2$ the saddle point is outside the integration range and the leading contribution to the integral comes from its lower limit. On easily shows that the integral vanishes for $V \rightarrow \infty$ and $\mu<m_{\pi} / 2$. For $\mu>m_{\pi} / 2$ the saddle point is inside the integration domain and its leading order exactly cancels the thermodynamic limit of the terms that are polynomial in $\mu$.

A similar essential singularity at $\mu=0$ is found for $N_{f}=2$, and the results obtained by Taylor expansion or analytic continuation in $\mu$ only reproduce the finite polynomial series in $\hat{\mu}^{2}$. What is not reproduced by analytic continuation are precisely the contributions given by the integrals in (47)-(49).

The integrals with the essential singularity are due to Dirac eigenvalues with real part larger than $m$. This is best illustrated by considering $X^{(0)}$ which is the partition function for one bosonic flavor [47]. In terms of a joint eigenvalue distribution it is given by

$$
Z_{N_{f}=-1}^{N}(m ; \mu)=\frac{1}{\mu^{2 N}} \int \prod_{k} d^{2} z_{k}\left|\Delta_{N}\left(\left\{z_{l}^{2}\right\}\right)\right|^{2} \prod_{k=1}^{N} w\left(z_{k}, z_{k}^{*} ; \mu\right) \frac{1}{\left(m^{2}-z_{k}^{2}\right)}
$$

where the Vandermonde determinant and the joint probability distribution are defined in (29) and (30), respectively. If we single out one eigenvalue, which we will denote by $w$ this can be re-expressed as

$$
Z_{N_{f}=-1}^{N}(m ; \mu)=\frac{N}{\mu^{2}} \int d^{2} w|w|^{2} K_{0}\left(\frac{N\left(1+\mu^{2}\right)}{2 \mu^{2}}|w|^{2}\right) \exp \left(-\frac{N\left(1-\mu^{2}\right)}{4 \mu^{2}}\left(w^{2}+w^{* 2}\right)\right) \frac{1}{\left(m^{2}-w^{2}\right)} Z_{N_{f}=1}^{N-1}\left(w^{*}\right),
$$

where we have used the identity [45]

$$
\prod_{k=1}^{N} \frac{1}{m^{2}-z_{k}^{2}}=\sum_{j=1}^{N} \frac{1}{m^{2}-z_{j}^{2}} \prod_{k \neq j}^{N} \frac{1}{z_{j}^{2}-z_{k}^{2}}
$$

Using that in the microscopic limit the partition function $Z_{N_{f}=1}^{N-1}\left(w^{*}\right)$ is given by

$$
Z_{N_{f}=1}^{N-1}\left(w^{*}\right) \rightarrow I_{0}\left(\hat{w}^{*}\right)
$$

we obtain for the microscopic limit of $Z_{N_{f}=-1}^{N}$ (which as before is denoted by $X^{(0)}$ using the same normalization)

$$
X^{(0)}(\hat{m} ; \hat{\mu})=\frac{1}{4 \pi} \frac{1}{\hat{\mu}^{2}} \int d^{2} \hat{w}|\hat{w}|^{2} \frac{1}{\left(\hat{m}^{2}-\hat{w}^{2}\right)} K_{0}\left(\frac{|\hat{w}|^{2}}{2 \hat{\mu}^{2}}\right) \exp \left(-\frac{\hat{w}+\hat{w}^{* 2}}{4 \hat{\mu}^{2}}\right) I_{0}\left(\hat{w}^{*}\right) .
$$

The expression for $X^{(0)}$ given in (47) is obtained by integration over the imaginary part of $\hat{w}$ by means of Cauchy's theorem and introducing the variable $u=2 \operatorname{Re}(\hat{w})-\hat{m}$. The integral over $u>\hat{m}$ in (47), thus corresponds to contributions with the real part of one of the eigenvalues larger than $m$. The same argument can be made for $X^{(k>0)}(\hat{m} ; \hat{\mu})$.

For $\hat{\mu}^{2}>\hat{m} / 2$ the probability that one of the eigenvalues has a real part larger than $m$ remains nonzero in the thermodynamic limit so that the non-analytic term becomes important. In the thermodynamic limit it exactly cancels the analytic term resulting in a vanishing average phase factor. In figure 2 we illustrate that the contribution of the non-analytic term (given by the difference between the upper and lower dashed curve) is important close to the critical value of $\mu$ and beyond. For comparison we also give the thermodynamic limit of the average phase factor (full curve).

The source of the non-analyticity is the inverse determinant of a non-hermitian operator. For example, for the partition function with one bosonic flavor with imaginary chemical potential the commutator term in the chiral Lagrangian vanishes resulting in a microscopic partition function that is independent of $\mu$. For real $\mu$ the bosonic determinant has to be regularized resulting in a $\mu$-dependent effective Lagrangian (see [47] for details), and a partition function that is $\mu$-dependent and therefore non-analytic at $\mu=0$.

The message we would like to convey is that the Taylor expansion in $\mu$ about zero of averages which involve an inverse quark determinant has zero radius of convergence. Also traces of the inverse of non hermitian operators lead to essential singularities at $\mu=0$ unless the inverse is compensated by a factor from the fermionic determinant. The simplest example is the quenched chiral condensate which is non-analytic in $\mu$ at $\mu=0$.

Note that the average phase factor in the phase quenched theory (11) does not involve averages of inverse determinants and is analytic in $\mu$ at $\mu=0$ as can easily be verified from (15). 


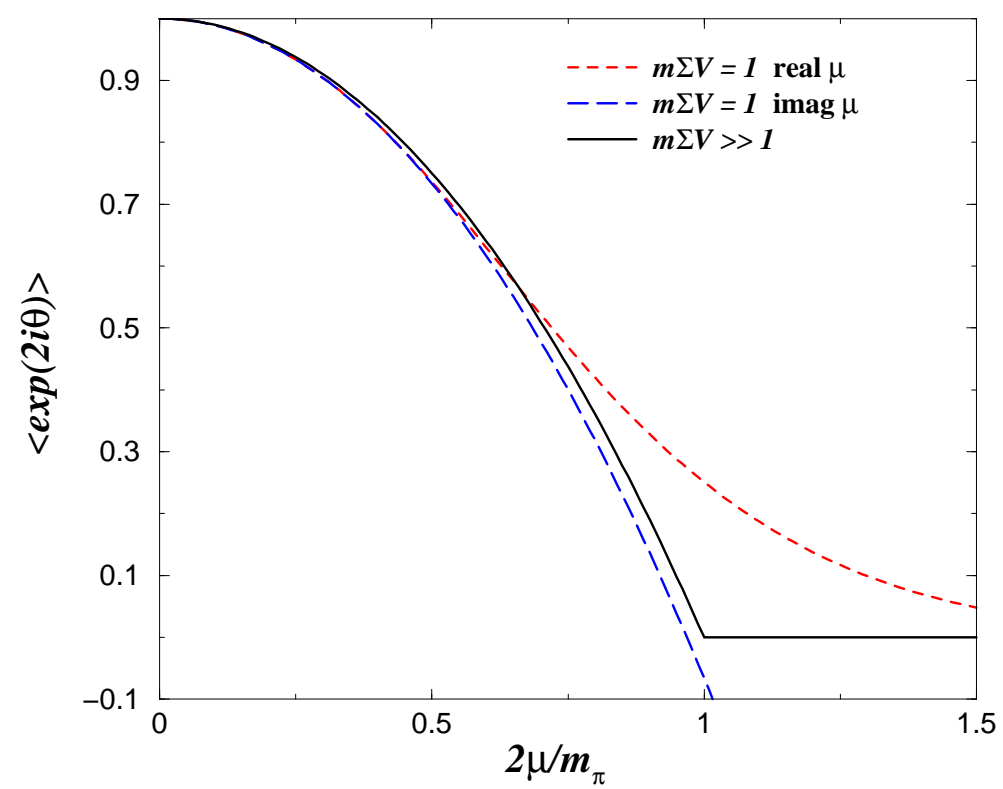

FIG. 2: The quenched average of the phase factor. Shown is the microscopic result for real $\mu$ (upper short dashed curve) and the microscopic result obtained by analytic continuation from imaginary chemical potential (lower long dashed curve). The full curve represents the thermodynamic result for real $\mu$. Note that while the average phase factor is non-analytic at $\mu=0$, the analytic continuation from imaginary $\mu$ follows closely the correct result for $2 \mu / m_{\pi} \ll 1$.

\section{1-LOOP CORRECTIONS}

So far we have focused on the average phase factor in the microscopic domain where $m_{\pi}^{2} F_{\pi}^{2} \sim 1 / V$ and $\mu^{2} F_{\pi}^{2} \sim 1 / V$ as the volume is taken to infinity. A perturbative expansion scheme where $p \sim 1 / L, m_{\pi} F_{\pi} \sim 1 / L^{2}, \mu F_{\pi} \sim 1 / L^{2}$ is known as the $\epsilon$-expansion [2]. The microscopic domain is the leading order term in the $\epsilon$-expansion. In this section we consider the so called $p$-expansion where $p \sim 1 / L, m_{\pi} \sim 1 / L, \mu \sim 1 / L$ and work out the average phase factor in an expansion in $m_{\pi}^{2} L^{2}$ and $\mu^{2} L^{2}$ for a finite but large box. In the $p$-expansion, the microscopic variables $\mu^{2} F_{\pi}^{2} V$ and $m \Sigma V$ are large, and consequently the leading term in the expansion is given by the asymptotic limit of the microscopic expression. Below we will compute subleading terms up to order $m_{\pi}^{2} \mu^{2} L^{4}$ and $\mu^{4} L^{4}$. This corresponds to the 1-loop corrections generated by the nonzero momentum terms of the Goldstone bosons. For simplicity we will consider only the average phase factor in the phase quenched theory for $\mu<m_{\pi} / 2$.

\section{A. 1-loop Integral at $\mu \neq 0$}

In the standard notation of chiral perturbation theory we have [49]

$$
\left\langle e^{2 i \theta}\right\rangle_{1+1^{*}}=\frac{Z_{N_{f}=2}}{Z_{1+1^{*}}}=\exp \left(G_{0}(\mu=0)-G_{0}(\mu)\right) .
$$

Notice that the contribution of the neutral Goldstone bosons cancels, and that we have a factor 2 in the exponent because the contribution of the two oppositely charged Goldstone bosons is the same. The 1-loop contributions of a single charged Goldstone boson with charge 2 ( $\mu$ is the quark chemical potential so a pion made from an up and a down quark has charge 2$)$ in a box with volume $L^{d}$ is given by

$$
e^{G_{0}(\mu) / 2} \equiv \exp \left[-\frac{1}{2} \sum_{p_{k \alpha}} \log \left(\vec{p}_{k}^{2}+m_{\pi}^{2}+\left(p_{k 0}-2 i \mu\right)^{2}\right)\right]
$$

where

$$
p_{k \alpha}=\frac{2 \pi k_{\alpha}}{L_{\alpha}}, \quad k_{\alpha} \quad \text { integer. }
$$


(For a fermionic Goldstone boson the sign of the exponent is positive.) The contribution to the free energy given by $G_{0}(\mu)$ is divergent. However, the difference between $G_{0}(\mu)$ and $G_{0}(\mu)$ for $V=\infty$ is finite, that is

$$
G_{0}(\mu)=\left.G_{0}(\mu)\right|_{V=\infty}+g_{0}(\mu)
$$

with $g_{0}(\mu)$ finite. Since $\left.G_{0}(\mu)\right|_{V=\infty}$ is independent of $\mu$ for $\mu<m_{\pi} / 2$ (in dimensional regularization, see e.g. [50]) we have that

$$
\left\langle e^{2 i \theta}\right\rangle_{1+1^{*}}=\frac{Z_{N_{f}=2}}{Z_{1+1^{*}}}=\exp \left(g_{0}(\mu=0)-g_{0}(\mu)\right)
$$

We wish to evaluate this 1 -loop contribution including the $1 / V$ corrections. This can be done along the lines of [49] where the expression for $g_{0}$ was worked out for $\mu=0$. The first step is to perform a Poisson resummation

$$
G_{0}(\mu)=-\sum_{p_{k \alpha}} \log \left(\vec{p}_{k}^{2}+m_{\pi}^{2}+\left(p_{k 0}-2 i \mu\right)^{2}\right)=-V \sum_{l_{\alpha}} \int \frac{d^{d} p}{(2 \pi)^{d}} e^{i L_{\alpha} p_{\alpha} l_{\alpha}} \log \left(\vec{p}^{2}+m_{\pi}^{2}+\left(p_{0}-2 i \mu\right)^{2}\right),
$$

where the sum is over all integers. The thermodynamic limit, $\left.G_{0}\right|_{V=\infty}$, is given by the term $l_{\alpha}=0$. Since this term does not contribute to $\langle\exp (2 i \theta)\rangle$ we exclude it from now on. For $\mu<m_{\pi} / 2$ we can shift the $p_{0}$ variable by $2 i \mu$. This results in

$$
\begin{aligned}
g_{0} & =-V \sum_{l_{\alpha}}^{\prime} \int \frac{d^{d} p}{(2 \pi)^{d}} e^{i L_{\alpha} p_{\alpha} l_{\alpha}-2 \mu l_{0} L_{0}} \log \left(\vec{p}^{2}+m_{\pi}^{2}+p_{0}^{2}\right) \\
& =-V \sum_{l_{\alpha}}^{\prime} \int \frac{d^{d} p}{(2 \pi)^{d}} e^{i L_{\alpha} p_{\alpha} l_{\alpha}-2 \mu l_{0} L_{0}} \lim _{\epsilon \rightarrow 0}\left[-\gamma+\frac{1}{\epsilon}-\int_{0}^{\infty} \frac{d \lambda}{\lambda^{1-\epsilon}} e^{\left.-\left(\vec{p}^{2}+m_{\pi}^{2}+p_{0}^{2}\right) \lambda\right]},\right.
\end{aligned}
$$

where $\gamma$ is the Euler constant. The $1 / \epsilon-\gamma$-terms do not contribute because the term with all $l_{\alpha}=0$ has been excluded from the sum. After performing the momentum integrals, the limit $\epsilon \rightarrow 0$ can be taken safely resulting in

$$
g_{0}=V \sum_{l_{\alpha}}^{\prime} \frac{1}{(2 \pi)^{d}} \pi^{d / 2} e^{-2 \mu l_{0} L_{0}} \int_{0}^{\infty} \frac{d \lambda}{\lambda} \lambda^{-d / 2} e^{-\frac{l_{\alpha}^{2} L_{\alpha}^{2}}{4 \lambda}} e^{-m_{\pi}^{2} \lambda} .
$$

Next we change to dimensionless integration variables by

$$
\lambda \rightarrow \lambda \frac{L^{2}}{4 \pi} \quad \text { with } \quad L=\left(\prod_{\alpha} L_{\alpha}\right)^{1 / d}
$$

and interchange the sums and the integral to arrive at

$$
g_{0}=\int_{0}^{\infty} \frac{d \lambda}{\lambda} \lambda^{-d / 2} e^{-m_{\pi}^{2} L^{2} \lambda / 4 \pi}\left(\prod_{\alpha} \sum_{l_{\alpha}} e^{-2 \mu l_{0} L_{0} \delta_{\alpha 0}} e^{-\pi \frac{l_{\alpha}^{2} L_{\alpha}^{2}}{\lambda L^{2}}}-1\right) .
$$

The integral over $\lambda$ can be split into into a part with $\lambda<1$ and a part with $\lambda>1$. On the second part we apply Jacobi's imaginary transformation

$$
\sum_{k=-\infty}^{\infty} e^{-a k^{2}-b k}=\sqrt{\frac{\pi}{a}} e^{\frac{b^{2}}{4 a}} \sum_{k=-\infty}^{\infty} e^{-\frac{\pi^{2} k^{2}}{a}-\frac{\pi i b k}{a}} .
$$

This leads to

$$
\begin{array}{r}
g_{0}=\int_{0}^{1} \frac{d \lambda}{\lambda} \lambda^{-d / 2} e^{-m_{\pi}^{2} L^{2} \lambda / 4 \pi}\left(\prod_{\alpha} \sum_{l_{\alpha}} e^{-2 \mu l_{0} L_{0} \delta_{\alpha 0}} e^{-\pi \frac{l_{\alpha}^{2} L_{\alpha}^{2}}{\lambda L^{2}}}-1\right) \\
+\int_{1}^{\infty} \frac{d \lambda}{\lambda} e^{\frac{\mu^{2} L^{2} \lambda}{\pi}} e^{-m_{\pi}^{2} L^{2} \lambda / 4 \pi}\left(\prod_{\alpha} \sum_{l_{\alpha}} e^{-2 i \mu l_{0} \frac{\lambda L^{2}}{L_{0}} \delta_{\alpha 0}} e^{-\pi l_{\alpha}^{2} \frac{\lambda L^{2}}{L_{\alpha}^{2}}}-1\right)+r_{0} .
\end{array}
$$

The Jacobi imaginary transformation is applied to the full sum so that the subtracted term before and after the transformation is different. This difference, denoted by $r_{0}$, is given by

$$
r_{0}=\int_{1}^{\infty} \frac{d \lambda}{\lambda} e^{\frac{\mu^{2} L^{2} \lambda}{\pi}} e^{-m_{\pi}^{2} L^{2} \lambda / 4 \pi}-\int_{1}^{\infty} \frac{d \lambda}{\lambda} \lambda^{-d / 2} e^{-m_{\pi}^{2} L^{2} \lambda / 4 \pi}
$$


Finally, we change $\lambda \rightarrow 1 / \lambda$ in the Jacobi transformed terms in $g_{0}$ to obtain

$$
\begin{array}{r}
g_{0}=\int_{0}^{1} \frac{d \lambda}{\lambda} \lambda^{-d / 2} e^{-m_{\pi}^{2} L^{2} \lambda / 4 \pi}\left(\prod_{\alpha} \sum_{l_{\alpha}} e^{-2 \mu l_{0} L_{0} \delta_{\alpha 0}} e^{-\pi \frac{l_{\alpha}^{2} L_{\alpha}^{2}}{\lambda L^{2}}}-1\right) \\
+\int_{0}^{1} \frac{d \lambda}{\lambda} e^{\frac{\mu^{2} L^{2}}{\pi \lambda}} e^{-m_{\pi}^{2} L^{2} /(4 \pi \lambda)}\left(\prod \sum_{\alpha} e^{-2 i \mu l_{0} \frac{L^{2}}{L_{0} \lambda} \delta_{\alpha 0}} e^{-\pi l_{\alpha}^{2} \frac{L^{2}}{L_{\alpha}^{2} \lambda}}-1\right)+r_{0} .
\end{array}
$$

The expansion of $r_{0}$ follows immediately from [49]. The only modification is that the Goldstone boson mass in one of the terms now depends on the chemical potential. We find

$$
\begin{aligned}
r_{0}= & -\log \frac{\left(m_{\pi}^{2}-4 \mu^{2}\right) L^{2}}{4 \pi}-\gamma-\sum_{n=1}^{\infty} \frac{1}{n ! n}\left(-\frac{\left(m_{\pi}^{2}-4 \mu^{2}\right) L^{2}}{4 \pi}\right)^{n} \\
& +\frac{1}{2}\left(\frac{m_{\pi}^{2} L^{2}}{4 \pi}\right)^{2}\left[\log \frac{m_{\pi}^{2} L^{2}}{4 \pi}+\gamma-\frac{3}{2}\right]-\frac{1}{2}+\frac{m_{\pi}^{2} L^{2}}{4 \pi}+\sum_{n=3}^{\infty} \frac{1}{n !(n-2)}\left(-\frac{m_{\pi}^{2} L^{2}}{4 \pi}\right)^{n} .
\end{aligned}
$$

For the difference $g_{0}-r_{0}$ we obtain the expansion

$$
g_{0}-r_{0}=\sum_{n=0}^{\infty} \sum_{m=0}^{\infty} \frac{1}{n !(2 m) !}\left[\left(-\frac{m_{\pi}^{2} L^{2}}{4 \pi}\right)^{n}\left(4 \mu^{2} L^{2}\right)^{m} S_{n-2, m}+\left(-\frac{\left(m_{\pi}^{2}-4 \mu^{2}\right) L^{2}}{4 \pi}\right)^{n}\left(-4 \mu^{2} L^{2}\right)^{m} S_{-n-2 m, m}\right] .
$$

For a $d$-dimensional hypercubic box the shape coefficients $S_{k, l}$ are given by

$$
\begin{aligned}
S_{k, l>0} & =\int_{0}^{1} \frac{d \lambda}{\lambda} \lambda^{k} \sum_{l_{0}} l_{0}^{2 l} e^{-\pi l_{0}^{2} / \lambda}\left(\sum_{l_{1}} e^{-\pi l_{1}^{2} / \lambda}\right)^{d-1}, \\
S_{k, 0} & =\int_{0}^{1} \frac{d \lambda}{\lambda} \lambda^{k}\left[\left(\sum_{l_{1}} e^{-\pi l_{1}^{2} / \lambda}\right)^{d}-1\right] .
\end{aligned}
$$

The shape coefficients $S_{k, 0}$ already enter in the expansion for $\mu=0$ [49]. In the table below we give the numerical value of a few low order coefficients. The lowest order terms of the expansion are given by

$$
\begin{aligned}
& \begin{array}{c|ccc}
S_{k, l} & l=0 & l=1 & l=2 \\
\hline k=0 & 0.09375685 & 0.025131706 & 0.025139306 \\
k=-1 & 0.11745759 & 0.031290174 & 0.031298318 \\
k=-2 & 0.15365038 & 0.040637153 & 0.040645920 \\
k=-3 & 0.21251023 & 0.055744392 & 0.055753879 \\
k=-4 & 0.31550608 & 0.082023256 & 0.082033581
\end{array} \\
& g_{0}=-\log \frac{\left(m_{\pi}^{2}-4 \mu^{2}\right) L^{2}}{4 \pi}-\gamma-\frac{1}{2}+S_{0,0}+S_{-2,0}+\frac{1}{2}\left(\frac{m_{\pi}^{2} L^{2}}{4 \pi}\right)^{2}\left[\log \frac{m_{\pi}^{2} L^{2}}{4 \pi}+\gamma-\frac{3}{2}\right] \\
& +\frac{\left(m_{\pi}^{2}-4 \mu^{2}\right) L^{2}}{4 \pi}\left(1-S_{-1,0}\right)+\frac{m_{\pi}^{2} L^{2}}{4 \pi}\left(1-S_{-1,0}\right) \\
& +\left(\frac{\left(m_{\pi}^{2}-4 \mu^{2}\right) L^{2}}{4 \pi}\right)^{2}\left(-\frac{1}{4}+\frac{1}{2} S_{-2,0}\right)+\left(\frac{m_{\pi}^{2} L^{2}}{4 \pi}\right)^{2} \frac{1}{2} S_{0,0} \\
& +\frac{m_{\pi}^{2} L^{2}}{8 \pi} 4 \mu^{2} L^{2}\left(-S_{-1,1}+S_{-3,1}\right)+\left(4 \mu^{2} L^{2}\right)^{2}\left(\frac{1}{24} S_{-2,2}+\frac{1}{24} S_{-4,2}-\frac{1}{8 \pi} S_{-3,1}\right) .
\end{aligned}
$$

The $\mu$ independent terms do not contribute to the average phase so that at next-to-next-to-leading order we find

$$
\begin{aligned}
\left\langle e^{2 i \theta}\right\rangle_{1+1^{*}}= & \left(1-\frac{4 \mu^{2}}{m_{\pi}^{2}}\right) \exp \left(\frac{4 \mu^{2} L^{2}}{4 \pi}\left(1-S_{-1,0}\right)+\frac{8 m_{\pi}^{2} \mu^{2} L^{4}-16 \mu^{4} L^{4}}{(4 \pi)^{2}}\left(-\frac{1}{4}+\frac{1}{2} S_{-2,0}\right)\right. \\
& \left.-\frac{m_{\pi}^{2} L^{2}}{8 \pi} 4 \mu^{2} L^{2}\left(-S_{-1,1}+S_{-3,1}\right)-\left(4 \mu^{2} L^{2}\right)^{2}\left(\frac{1}{24} S_{-2,2}+\frac{1}{24} S_{-4,2}-\frac{1}{8 \pi} S_{-3,1}\right)\right)
\end{aligned}
$$




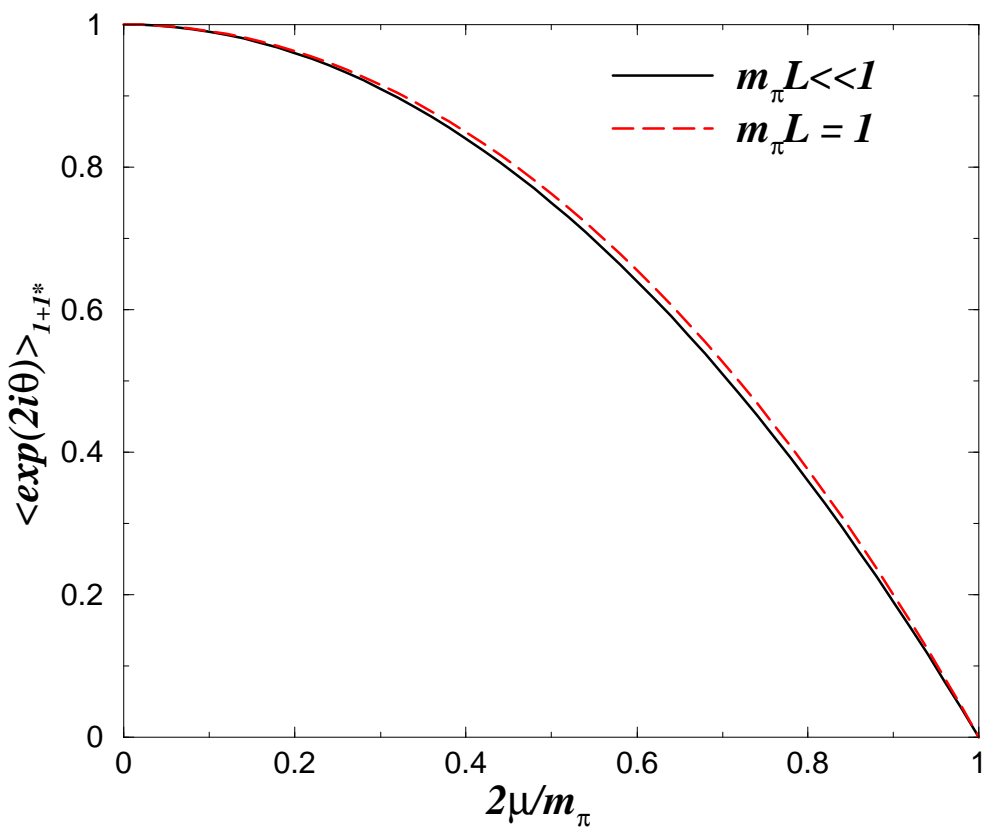

FIG. 3: The average phase factor in the phase quenched theory as a function of the chemical potential in the normal phase. The full line gives the result at leading order (21) and the dashed line includes the corrections up to next-to-next-to-leading order (92) when $m_{\pi} L=1$.

The $\mu L \rightarrow 0, m_{\pi} L \rightarrow 0$ limit of this expression should reproduce the $\hat{\mu} \rightarrow \infty, \hat{m} \rightarrow \infty$ limit of the microscopic result which is indeed the case (see (21)). The correction factor computed in (92) drives the average phase factor closer to 1 (see figure 3). Note that the correction is small even though $m_{\pi} L$ is set to 1 .

\section{CONCLUSIONS}

We have computed the average phase factor in the microscopic domain of quenched, unquenched as well as phase quenched QCD and have found that in the thermodynamic limit the average phase factor undergoes a phase transition at $\mu=m_{\pi} / 2$. For $\mu<m_{\pi} / 2$ it falls off polynomially with $\mu$ and is equal to zero starting from $\mu=m_{\pi} / 2$. This implies that the phase problem becomes severe for $\mu>m_{\pi} / 2$. Physically this should be the case because the phase factor has to wipe out the Bose condensate that would be the vacuum state if the fermion determinant would have been replaced by its absolute value.

The results for the average phase factor in the microscopic limit of QCD were derived from a chiral random matrix model exploiting the equivalence of these two theories in the microscopic domain. Powerful random matrix methods such as the method of orthogonal polynomials and their Cauchy transforms enabled us to obtain exact analytical results. The thermodynamic limit of these exact results, was also derived from simple mean field arguments. The starting point was that the average phase factor is the inverse ratio of the QCD partition function and the partition function with the fermion determinant of QCD and an additional conjugate bosonic quark and a fermionic quark. For $\mu<m_{\pi} / 2$ the partition function with the additional quarks is in the same phase as the partition function without additional quarks. The ratio of the two partition functions is therefore determined by the $1 / V$ corrections to the free energies. For larger values of $\mu$ the presence of the conjugate bosonic quark induces a pion condensate and the two free energies no longer cancel in the thermodynamic limit. This causes the exponential suppression with the volume of the average phase factor.

While the average phase factor is exponentially small for $\mu>m_{\pi} / 2$ the fluctuations of the phase factor becomes unity for $\mu>m_{\pi} / 2$. The sign problem is therefore particularly severe for $\mu>m_{\pi} / 2$.

The finiteness of the average phase factor for $\mu<m_{\pi} / 2$ suggests that it should be possible to test the predictions of this paper by means of lattice QCD simulations. We note however that the quenched and unquenched phase factor is non-analytic in $\mu$ at $\mu=0$. As has been shown explicitly in the quenched case, the phase factor as defined by the ratio of two determinants with opposite sign of the chemical potential, cannot be analytically continued from negative $\mu^{2}$ to positive $\mu^{2}$. It is our experience that analyticity at $\mu=0$ is lost for averages involving the inverse (determinant) 
of the nonhermitian Dirac operator when the singularity is not compensated by a similar factor from the fermion determinant. In such cases lattice methods that rely on analyticity cannot be used. On the other hand, we expect observables that are derivatives of the usual fermionic partition functions to be are analytic in $\mu$ for $\mu \rightarrow 0$ and can be computed by means of the Taylor expansion method or the imaginary chemical potential method.

Since each eigenvalue contributes to the phase factor it is perhaps surprising that the average phase factor is not sensitive to the ultra-violet cutoff. The reason goes back to the renormalizablity of chiral perturbation theory: If the theory is renormalized at $\mu=0$, the $\mu$ dependence does not introduce any additional infinities. Since the average phase factor is a partition function at $\mu \neq 0$ divided by the same partition function $\mu=0$, it is therefore necessarily ultraviolet finite. We have shown this explicitly to 1-loop order for the phase quenched partition function. This follows from the microscopic theory as well. In lattice QCD the Hasenfratz-Karsch prescription [51] to introduce the chemical potential does not give rise to additional ultraviolet divergences in lattice perturbation theory. However, nonperturbatively the situation on the lattice is less clear. Potentially dangerous ultraviolet contributions may appear for a naive evaluation of the phase factor of the fermion determinant on the lattice. Despite of these and other potential pitfalls, it is our hope that the results of this paper can be compared to lattice QCD and contribute constructively to our understanding of QCD at finite density.

Acknowledgments. We wish to thank S. Hands, C. Allton, G. Akemann, P.H. Damgaard, P. de Forcrand, M. Lüscher, D. Dietrich, J. Osborn and L. Ravagli for valuable discussions. This work was supported by U.S. DOE Grant No. DE-FG-88ER40388 (JV), the Carlsberg Foundation (KS), the Villum Kann Rassmussen Foundation (JV) and the Danish National Bank (JV). 


\section{APPENDIX A: PARAMETERIZATION INDEPENDENCE OF MEAN FIELD RESULTS.}

In this appendix we illustrate that the mean field result including the 1-loop fluctuations about the saddle point (see eq. (7D) does not depend on the parametrization of the integration manifold. The nontrivial example we study is the phase quenched partition function in the condensed phase, i.e. for $\mu>m_{\pi} / 2$. Because of the presence of an exactly massless mode in this phase, we include a pion source which explicitly breaks the $U(1)$ symmetry that is responsible for the massless mode. This allows us to use the saddle point approximation to show that the partition function is independent of the representation whereas the Jacobian and masses are representation dependent.

The pion condensate source term enters in the mass matrix

$$
\left(\begin{array}{cc}
m & 0 \\
0 & m
\end{array}\right) \rightarrow\left(\begin{array}{cc}
m & \lambda \\
-\lambda & m
\end{array}\right)
$$

so that the otherwise massless mode obtains a mass $\sim \sqrt{\lambda}$. We will consider two different representations of the Goldstone fields

$$
U=\left(\begin{array}{cc}
\cos \theta e^{i \alpha} & e^{i \phi} \sin \theta \\
-e^{-i \phi} \sin \theta & \cos \theta e^{-i \alpha}
\end{array}\right) e^{i \omega} \quad \text { and } \quad U=e^{i \bar{\theta} \tau_{1}} e^{i \pi_{k} \tau_{k}} e^{i \omega}
$$

where $\bar{\theta}$ is a vacuum angle that will be determined by the saddle point equations. The Pauli matrices are labeled $\tau_{k}$. The partition function in either variables is given by $(\hat{\lambda}=\lambda \Sigma V)$

$$
Z_{1+1^{*}}^{\mathrm{A}}(\hat{m}, \hat{\lambda} ; \hat{\mu})=\int d \theta d \alpha d \phi d \omega \sin \theta \cos \theta e^{2 \hat{\mu}^{2} \sin ^{2} \theta+2 \hat{m} \cos \theta \cos \alpha \cos \omega+2 \hat{\lambda} \sin \theta \cos \phi \cos \omega},
$$

and

$$
\begin{aligned}
Z_{1+1^{*}}^{\mathrm{B}}(\hat{m}, \hat{\lambda} ; \hat{\mu})=\int & d \pi_{1} d \pi_{2} d \pi_{3} d \omega \frac{\sin ^{2} \pi}{\pi^{2}} e^{\hat{\mu}^{2}\left[\left(\cos \bar{\theta} \frac{\sin \pi}{\pi} \pi_{1}+\cos \pi \sin \bar{\theta}\right)^{2}+\left(\cos \overline{\bar{\theta}} \frac{\sin \pi}{\pi} \pi_{2}+\sin \bar{\theta} \frac{\sin \pi}{\pi} \pi_{3}\right)^{2}\right]} \\
& \times e^{2 \hat{m}\left[\cos \bar{\theta} \cos \pi-\sin \bar{\theta} \frac{\sin \pi}{\pi} \pi_{1}\right] \cos \omega+\hat{\lambda}\left[\cos \bar{\theta} \frac{\sin \pi}{\pi} \pi_{1}+\cos \pi \sin \bar{\theta}\right] \cos \omega}
\end{aligned}
$$

where $\pi=\sqrt{\pi_{1}^{2}+\pi_{2}^{2}+\pi_{3}^{2}}$. We will evaluate both partition functions in the thermodynamic limit, $\hat{\mu}^{2} \rightarrow \infty, \hat{m} \rightarrow \infty$ and $\hat{\lambda} \rightarrow \infty$, where the integrals can be performed by a saddle point approximation. The parameter $\bar{\theta}$ will be chosen such that the saddle point of the $\pi_{k}$ variables is at zero. It turns out that this is also the saddle point of the $\theta$-integral in (A3). In terms of the $\theta-\alpha-\phi-\omega$ variables the squared masses are given by

$$
\hat{m} \cos \bar{\theta}, \quad \hat{\lambda} \sin \bar{\theta}, \quad 2 \hat{\mu}^{2} \sin ^{2} \bar{\theta}+\frac{\hat{\lambda}}{\sin \bar{\theta}}, \quad \hat{m} \cos \bar{\theta}+\hat{\lambda} \sin \bar{\theta}
$$

with $\bar{\theta}$ determined by the saddle point equation

$$
2 \hat{\mu}^{2} \cos \bar{\theta} \sin \bar{\theta}-\hat{m} \sin \bar{\theta}+\hat{\lambda} \cos \bar{\theta}=0 .
$$

The squared masses in terms $\pi_{k}-\omega$-variables can be rewritten by using the saddle point equation which is also given by (A6). We find

$$
2 \hat{\mu}^{2}+\frac{\hat{\lambda}}{\sin \bar{\theta}}=\frac{\hat{m}}{\cos \bar{\theta}}, \quad \frac{\hat{\lambda}}{\sin \bar{\theta}}, \quad 2 \hat{\mu}^{2} \sin ^{2} \bar{\theta}+\frac{\hat{\lambda}}{\sin \bar{\theta}}, \quad \hat{m} \cos \bar{\theta}+\hat{\lambda} \sin \bar{\theta}
$$

The product of the squared masses is different for the two parameterizations. The contribution from the Jacobian in (A3), $\sin \bar{\theta} \cos \bar{\theta}$ exactly compensates for this difference such that the mean field partition function (7) is identical in the two cases. In the limit of $\hat{\lambda} \ll \hat{m}$ and $\hat{\lambda} \ll \hat{\mu}^{2}$ we obtain

$$
Z_{1+1^{*}}(\hat{m}, \hat{\lambda} ; \hat{\mu})=\frac{\pi^{2}}{\hat{m} \sqrt{\hat{\lambda}} \sqrt{2 \hat{\mu}^{2}-\hat{m}^{2} / 2 \hat{\mu}^{2}}} e^{2 \hat{\mu}^{2}+\hat{m}^{2} / 2 \hat{\mu}^{2}},
$$

in agreement with the general expression (7).

What we have learned from this example is that the chemical potential dependence of the partition function originates both from both the masses and the Jacobian with neither of them being representation independent. 


\section{APPENDIX B: INTEGRALS APPEARING IN CAUCHY TRANSFORMS}

In this appendix we evaluate some integrals that appear in the Cauchy transforms $X^{(k)}$ given in (46).

To simplify the integrals appearing in $X^{(k)}$ the following integral is useful

$$
\int_{0}^{\infty} d t t e^{-p^{2} t^{2}}\left[K_{0}(a t) I_{0}(b t)+I_{0}(a t) K_{0}(b t)\right]=\frac{1}{2 p^{2}} e^{\frac{a^{2}+b^{2}}{4 p^{2}}} K_{0}\left(\frac{a b}{2 p^{2}}\right) .
$$

This identity can be proved by considering the integral

$$
\int_{0}^{\infty} d t t e^{-p^{2} t^{2}}\left[K_{0}(a t) I_{0}(b t)+I_{0}(a t) K_{0}(b t)\right]=\lim _{\nu \rightarrow 0} \int_{0}^{\infty} d t t e^{-p^{2} t^{2}}\left[K_{\nu}(a t) I_{\nu}(b t)+I_{-\nu}(a t) K_{-\nu}(b t)\right],
$$

and using the identity

$$
K_{\nu}(x)=\frac{\pi}{2} \frac{I_{-\nu}(x)-I_{\nu}(x)}{\sin (\nu \pi)}
$$

to replace the $K_{ \pm \nu}$ function by a $I_{ \pm \nu}$ functions.

By differentiation with respect to the parameters of this integral, we can derive the following useful identities:

$$
\begin{gathered}
\int_{0}^{\infty} d t t^{2} e^{-p^{2} t^{2}}\left[K_{0}(a t) I_{1}(b t)-I_{0}(a t) K_{1}(b t)\right]=\frac{1}{4 p^{4}} e^{\frac{a^{2}+b^{2}}{4 p^{2}}}\left[b K_{0}\left(\frac{a b}{2 p^{2}}\right)-a K_{1}\left(\frac{a b}{2 p^{2}}\right)\right], \\
\int_{0}^{\infty} d t t^{3} e^{-p^{2} t^{2}}\left[K_{0}(a t) I_{0}(b t)+I_{0}(a t) K_{0}(b t)\right]=\frac{1}{4 p^{4}} e^{\frac{a^{2}+b^{2}}{4 p^{2}}}\left[\left(2+\frac{a^{2}+b^{2}}{2 p^{2}}\right) K_{0}\left(\frac{a b}{2 p^{2}}\right)-\frac{a b}{p^{2}} K_{1}\left(\frac{a b}{2 p^{2}}\right)\right], \\
\int_{0}^{\infty} d t t^{4} e^{-p^{2} t^{2}}\left[K_{0}(a t) I_{1}(b t)-I_{0}(a t) K_{1}(b t)\right]= \\
\frac{1}{8 p^{6}} e^{\frac{a^{2}+b^{2}}{4 p^{2}}}\left[\left(4 b+\frac{b\left(3 a^{2}+b^{2}\right)}{2 p^{2}}\right) K_{0}\left(\frac{a b}{2 p^{2}}\right)-\left(2 a+\frac{a\left(a^{2}+3 b^{2}\right)}{2 p^{2}}\right) K_{1}\left(\frac{a b}{2 p^{2}}\right)\right] .
\end{gathered}
$$

\section{APPENDIX C: ASYMPTOTIC EXPANSION OF INTEGRALS OCCURRING IN THE $X^{(k)}$}

In this section we calculate the asymptotic expansion of the integrals occurring in the $X^{(k)}$ given in eq. (46). We separately consider the integral

$$
S_{p, \nu}=\int_{0}^{\hat{m}} d x x^{p} \exp \left[-\frac{x^{2}}{8 \hat{\mu}^{2}}\right] K_{0}\left(\frac{x \hat{m}}{4 \hat{\mu}^{2}}\right) I_{\nu}(x)
$$

in the normal phase and in the condensed phase.

In the normal phase, for $\hat{m} / 4<\hat{\mu}^{2}<\hat{m} / 2$, the saddle point at $\bar{x}=4 \hat{\mu}^{2}-\hat{m}$ of the integral (C1) is inside the integration domain. For $\hat{m} \rightarrow \infty$ and $\hat{\mu}^{2} \rightarrow \infty$ the Bessel functions can be expanded to leading order resulting in

$$
S_{p, \nu}^{\text {normal }}=4 \sqrt{\frac{\pi}{2 \hat{m}}} \hat{\mu}^{2}\left(4 \hat{\mu}^{2}-\hat{m}\right)^{p-1} e^{2 \hat{\mu}^{2}-\hat{m}+\frac{\hat{m}^{2}}{8 \hat{\mu}^{2}}}
$$

For $\hat{\mu}^{2}<\hat{m} / 4$ the leading saddle point contribution comes from the second integral in the $X^{(k)}$. We leave it up to the reader to show that the leading order saddle point approximation results in the same expression.

In the condensed phase the leading order expansion of $S_{p, \nu}$ cancels in the expression for the average phase. Therefore we have to include the subleading terms in its asymptotic expansion. Including the expansion of the Bessel functions to subleading order we obtain for $S_{p, \nu}$

$$
S_{p, \nu}^{\text {condensed }} \sim \sqrt{\frac{\hat{\mu}^{2}}{\hat{m}}} \int_{0}^{\hat{m}} d x x^{p-1} e^{-x^{2} / 8 \hat{\mu}^{2}-x \hat{m} / 4 \hat{\mu}^{2}+x}\left[1-\frac{\hat{\mu}^{2}}{2 x \hat{m}}-\frac{4 \nu^{2}-1}{8} \frac{1}{x}\right] .
$$

Next put $x=\hat{m}-t$. Since the integral is dominated by the vicinity of $t=0$ we can safely extend the integration range to $\infty$,

$$
S_{p, \nu}^{\text {condensed }} \sim \sqrt{\frac{\hat{\mu}^{2}}{\hat{m}}} e^{\hat{m}-\frac{3 \hat{m}^{2}}{8 \hat{\mu}^{2}}} \int_{0}^{\infty} d t\left(\hat{m}^{p-1}-(p-1) \hat{m}^{p-2} t\right) e^{-t\left(1-\frac{\hat{m}}{2 \hat{\mu}^{2}}\right)-\frac{t^{2}}{8 \hat{\mu}^{2}}}\left[1-\frac{\hat{\mu}^{2}}{2 \hat{m}^{2}}-\frac{4 \nu^{2}-1}{8} \frac{1}{\hat{m}}\right]
$$


To leading nonvanishing order it suffices to expand $\exp \left(-t^{2} / 8 \hat{\mu}^{2}\right)$ as $1-\frac{t^{2}}{8 \hat{\mu}^{2}}$. After performing the integral over $t$ we find

$$
S_{p, \nu}^{\text {condensed }}=\sqrt{\frac{\hat{\mu}^{2}}{\hat{m}}} e^{\hat{m}-\frac{3 \hat{m}^{2}}{8 \hat{\mu}^{2}}} \hat{m}^{p-2}\left[\frac{2 \hat{m}}{2-\frac{\hat{m}}{\hat{\mu}^{2}}}-\frac{4(p-1)}{\left(2-\frac{\hat{m}}{\hat{\mu}^{2}}\right)^{2}}-\frac{2 \hat{m}}{\hat{\mu}^{2}\left(2-\hat{m} / \hat{\mu}^{2}\right)^{3}}-\frac{1}{\left(2-\hat{m} / \hat{\mu}^{2}\right)}\left[\frac{\hat{\mu}^{2}}{\hat{m}}+\frac{4 \nu^{2}-1}{4}\right]\right] .
$$

Combining the different terms we obtain for the thermodynamic limit of the expectation value of the quenched phase

$$
\langle\exp (2 i \theta)\rangle_{N_{f}=0}=\frac{1}{\sqrt{2 \pi \hat{m}}} \frac{1}{\hat{\mu}^{2}\left(2-\hat{m} / \hat{\mu}^{2}\right)^{2}} \sqrt{\frac{\hat{\mu}^{2}}{\hat{m}}} e^{2 \hat{m}-\hat{m} / 2 \hat{\mu}^{2}-2 \hat{\mu}^{2}} \quad \text { for } \quad 1 \ll \hat{m}<2 \hat{\mu}^{2} .
$$

The condition for the validity of the derivation of (C6) is that $\left(2 \hat{\mu}^{2}-\hat{m}\right) / \hat{\mu} \gg 1$, which is only violated very close to the critical point because the natural magnitude of this ratio of $O(\sqrt{V})$. We can also evaluate the integral (C4) when this condition is not satisfied. Then the integral (C4) is cut-off by the the Gaussian factor $\exp \left(-t^{2} / 2 \hat{\mu}^{2}\right)$ instead of the exponential factor resulting in an expression that remains finite for $m=2 \mu^{2}$.

[1] I. M. Barbour, S. E. Morrison, E. G. Klepfish, J. B. Kogut and M. P. Lombardo, Nucl. Phys. Proc. Suppl. 60 A (1998) 220 .

[2] J. Gasser and H. Leutwyler, Phys. Lett. B 188, 477 (1987).

[3] H. Leutwyler and A. Smilga, Phys. Rev. D 46, 5607 (1992).

[4] Z. Fodor and S. D. Katz, JHEP 0203, 014 (2002) arXiv:hep-lat/0106002.

[5] Z. Fodor and S. D. Katz, JHEP 0404, 050 (2004) arXiv:hep-lat/0402006.

[6] P. de Forcrand and O. Philipsen, Nucl. Phys. B 642, 290 (2002) arXiv:hep-lat/0205016].

[7] P. de Forcrand and O. Philipsen, Nucl. Phys. B 673, 170 (2003) arXiv:hep-lat/0307020.

[8] M. D'Elia and M. P. Lombardo, Phys. Rev. D 67, 014505 (2003) arXiv:hep-lat/0209146.

[9] C. R. Allton et al., Phys. Rev. D 66, 074507 (2002) arXiv:hep-lat/0204010.

[10] C. R. Allton, S. Ejiri, S. J. Hands, O. Kaczmarek, F. Karsch, E. Laermann and C. Schmidt, Phys. Rev. D 68, 014507 (2003) arXiv:hep-lat/0305007.

[11] C. R. Allton et al., Phys. Rev. D 71, 054508 (2005) arXiv:hep-lat/0501030.

[12] R. V. Gavai and S. Gupta, Phys. Rev. D 68, 034506 (2003) arXiv:hep-lat/0303013.

[13] V. Azcoiti, G. di Carlo and A. F. Grillo, Phys. Rev. Lett. 65, 2239 (1990); J. Ambjorn, K. N. Anagnostopoulos, J. Nishimura and J. J. M. Verbaarschot, JHEP 0210, 062 (2002) arXiv:hep-lat/0208025; C. Schmidt, Z. Fodor and S. D. Katz, arXiv:hep-lat/0512032.

[14] C. Schmidt, PoS LAT2006 021, arXiv:hep-lat/0610116.

[15] D. Toussaint, Nucl. Phys. Proc. Suppl. 17, 248 (1990).

[16] P. de Forcrand and V. Laliena, Phys. Rev. D 61, 034502 (2000).

[17] Y. Sasai, A. Nakamura and T. Takaishi, Nucl. Phys. Proc. Suppl. 129, 539 (2004).

[18] S. Ejiri, Phys. Rev. D 69, 094506 (2004).

[19] S. Ejiri, Phys. Rev. D 73, 054502 (2006).

[20] P. E. Gibbs, PRINT-86-0389 (GLASGOW); Phys. Lett. B 182 (1986) 369.

[21] J. B. Kogut, H. Matsuoka, M. Stone, H. W. Wyld, S. H. Shenker, J. Shigemitsu and D. K. Sinclair, Nucl. Phys. B 225, 93 (1983); I. Barbour, N. E. Behilil, E. Dagotto, F. Karsch, A. Moreo, M. Stone and H. W. Wyld, Nucl. Phys. B 275, 296 (1986);

[22] M. Alford, A. Kapustin, and F. Wilczek, Phys. Rev. D 59 (1999) 054502.

[23] J.B. Kogut, M.A. Stephanov, and D. Toublan, Phys. Lett. B 464, 183 (1999).

[24] J.B. Kogut, M.A. Stephanov, D. Toublan, J.J.M. Verbaarschot, and A. Zhitnitsky, Nucl. Phys. B 582, 477 (2000).

[25] D. T. Son and M. A. Stephanov, Phys. Rev. Lett. 86, 592 (2001).

[26] K. Splittorff, D. T. Son, and M. A. Stephanov, Phys. Rev. D 64, 016003 (2001); J. B. Kogut and D. Toublan, Phys. Rev. D 64, 034007 (2001); J.T. Lenaghan, F. Sannino, K. Splittorff, Phys. Rev. D 65, 054002 (2002) J. Wirstam, J. T. Lenaghan, K. Splittorff, Phys. Rev. D 67, 034021 (2003); G. V. Dunne and S. M. Nishigaki, Nucl. Phys. B 654, 445 (2003); G. V. Dunne and S. M. Nishigaki, Nucl. Phys. B 670, 307 (2003); T. Brauner, Mod. Phys. Lett. A 21, 559 (2006).

[27] M. Stephanov, Phys. Rev. Lett. 76, 4472 (1996).

[28] V.L. Girko, Theory of random determinants (Kluwer Academic Publishers, Dordrecht, 1990).

[29] M. P. Lombardo, J. B. Kogut and D. K. Sinclair, Phys. Rev. D 54, 2303 (1996).

[30] K. Splittorff, PoS LAT2006 023, arXiv:hep-lat/0610072

[31] G. Akemann, J. C. Osborn, K. Splittorff and J. J. M. Verbaarschot, Nucl. Phys. B 712, 287 (2005).

[32] J. C. Osborn, Phys. Rev. Lett. 93, 222001 (2004).

[33] J. C. Osborn, K. Splittorff and J. J. M. Verbaarschot, Phys. Rev. Lett. 94, 202001 (2005). 
[34] E. V. Shuryak and J. J. M. Verbaarschot, Nucl. Phys. A 560, 306 (1993).

[35] J. J. M. Verbaarschot, Phys. Rev. Lett. 72, 2531 (1994).

[36] J. J. M. Verbaarschot, Phys. Lett. B 368, 137 (1996).

[37] K. Splittorff and J. J. M. Verbaarschot, arXiv:hep-lat/0609076

[38] K. Splittorff and J. J. M. Verbaarschot, Nucl. Phys. B 683, 467 (2004).

[39] J. J. M. Verbaarschot and T. Wettig, Ann. Rev. Nucl. Part. Sci. 50, 343 (2000) arXiv:hep-ph/0003017.

[40] Y.V. Fyodorov, B. Khoruzhenko and H.J. Sommers, Ann. Inst. Henri Poincaré: Phys. Theor. 68, 449 (1998).

[41] G. Akemann, Phys. Rev. Lett. 80, 072002 (2002) hep-th/0204068; J. Phys. A: Math. Gen. 36, 3363 (2003) hep-th/0204246.

[42] G. Akemann, Y. V. Fyodorov and G. Vernizzi, Nucl. Phys. B 694, 59 (2004).

[43] M.C. Bergère, hep-th/0311227.

[44] M.C. Bergère, hep-th/0404126.

[45] G. Akemann and A. Pottier, J. Phys. A 37, L453 (2004).

[46] J.C. Osborn, K. Splittorff and J.J.M. Verbaarschot, in preparation.

[47] K. Splittorff and J. J. M. Verbaarschot, Nucl. Phys. B 757, 259 (2006).

[48] P. H. Damgaard, J. C. Osborn, D. Toublan and J. J. M. Verbaarschot, Nucl. Phys. B 547, 305 (1999).

[49] P. Hasenfratz and H. Leutwyler, Nucl. Phys. B 343, 241 (1990).

[50] K. Splittorff, D. Toublan, and J.J.M. Verbaarschot, Nucl. Phys. B 620, 290 (2002); Nucl. Phys. B 639, 524 (2002).

[51] P. Hasenfratz and F. Karsch, Phys. Lett. B 125, 308 (1983).

[52] R. A. Janik, M. A. Nowak, G. Papp and I. Zahed, Phys. Rev. Lett. 77, 4876 (1996) arXiv:hep-ph/9606329; R. A. Janik, M. A. Nowak, G. Papp, J. Wambach and I. Zahed, Phys. Rev. E 55, 4100 (1997); R. A. Janik, M. A. Nowak, G. Papp and I. Zahed, Nucl. Phys. B 501, 603 (1997).

[53] J. Feinberg and A. Zee, Nucl. Phys. B504, 579 (1997).

[54] In order to write the denominator of the $\langle\ldots\rangle$ as a bosonic integral, it has to be "hermiticized" [52, 53] which requires the introduction of an additional bosonic and fermionic determinant. Since the contribution of the additional fermion and boson cancels trivially in the mean field argument we can safely ignore this.

[55] This result was obtained in collaboration with P.H. Damgaard. To derive it one adds $-\mu^{2} F_{\pi}^{2} V / 4 \operatorname{Str}\left[\sigma_{3}, U\right]\left[\sigma_{3}, U^{-1}\right]$ to the action in section 4 of [48] and computes the partition function for equal masses. 